\title{
Measurement Foundations for Multiattribute Psychophysical Theories Based on First Order Polynomials
}

\author{
John M. MiYAmoto \\ University of Michigan, Ann Arbor, Michigan 48104
}

\begin{abstract}
The class of first order polynomial measurement representations is defined, and a method for proving the existence of such representations is described. The method is used to prove the existence of first order polynomial generalizations of expected utility theory, difference measurement, and additive conjoint measurement. It is then argued that first order polynomial representations provide a deep and far reaching characterization of psychological invariance for subjective magnitudes of multiattributed stimuli. To substantiate this point, two applications of first order polynomial representation theory to the foundations of psychophysics are described. First, Relation theory, a theory of subjective magnitude proposed by Shepard (Journal of Mathemutical Psychology, 1981, 24, 21-57) and Krantz (Journal of Mathematical Psychology, 1972, 9, 168-199), is generalized to a theory of magnitude for multiattributed stimuli. The generalization is based on a postulate of context invariance for the constituent uniattribute magnitudes of a multiattribute magnitude. Second, the power law for subjective magnitude is generalized to a multiattribute version of the power law. Finally, it is argued that a common logical pattern underlies multiattribute generalizations of psychological theories to first order polynomial representations. This abstract pattern suggests a strategy for theory construction in multiattribute psychophysics.
\end{abstract}

Circa 1970, Ralph Keeney and Howard Raiffa showed how utility representations could be generalized to a broad class of combination rules called quasi-additive, quasi-separable, or multilinear utility functions (Keeney, 1968, 1971, 1972; Keeney \& Raiffa, 1976; Raiffa, 1969). This class of utility representations can be defined as the class of real valued functions determined by some polynomial in arbitrarily many variables, none of which is raised to a power higher than one. Essentially, Keeney and Raiffa found that a utility function for a multiattributed set of consequences could be extended to a multilinear representation whenever every attribute satisfied a property called utility independence with respect to all remaining attributes. An analogous generalization of difference measurement was achieved more recently by

The research reported here was supported in part by PHS-training Grant GM-01231 from the National Institute of Health to the University of Michigan. I would like to thank Clyde Coombs. David Krantz, and J. Frank Yates for much valuable criticism and discussion. I would also like to thank the Editor, A. A. J. Marley, and an anonymous referee for some very helpful suggestions with regard to the exposition of this material. Address reprint requests to John M. Miyamoto, Department of Psychology, University of Michigan, Ann Arbor, Michigan 48104-1346. 
Dyer and Sarin (1979), working in the theory of riskless value, when they showed that the property of utility independence could be reformulated in the framework of the positive difference structure.

Although the importance of this work to theoretical and practical decision making is evident, it has not yet been recognized that it suggests a new and powerful approach to the psychophysics of subjective magnitude for multiattributed stimuli. This essay show how utility-inspired developments in polynomial measurement can be interpreted psychophysically as a natural generalization of relational theories of subjective magnitude proposed by Krantz (1972) and Shepard (1981). The power law for subjective magnitude will then be generalized to multiattributed stimuli. These generalizations will be found to depend on a characterization of context invariant psychophysical judgments.

The essay is organized into five sections. Section I defines the basic formalism for a class of measurement representations called first order polynomial representations. Section II applies this formalism to three basic measurements structures, utility measurement, difference measurement, and additive conjoint measurement. The emphasis here will be on abstract similarities among the generalizations of these theories to first order polynomial representations. In Section III, a theory of subjective magnitude for multiattributed stimuli will be presented that generalizes Relation theory, a theory of subjective magnitude proposed by Shepard (1981) and Krantz (1972). Section IV describes a multiattribute generalization of the power law for subjective magnitude. Finally, it is argued in Section $V$ that first order polynomial measurement provides a general methodology for the formulation of multiattribute psychophysical theories. The fundamental, substantive construct of this approach is an abstract characterization of context invariance for psychophysical judgment.

\section{First Order Polynomials}

This section presents an abstract characterization of first order polynomial representations. Material drawn from the algebraic theory of polynomials (cf. MacLane \& Birkhoff, 1967) and the foundations of measurement (cf. Krantz, Luce, Suppes, \& Tversky, 1971) is assumed to be familiar. The principal mathematical properties of first order polynomial representations are stated in subsection I(A) while proofs establishing these properties are given in subsection $I(B)$. The proofs can be skipped since an understanding of them is not presupposed anywhere in the essay.

\section{I(A). Representation Theory}

By a first order polynomial in $N$ variables, we mean a real polynomial in $N$ variables in which no variable is raised to a power other than 0 or 1 . For example, the general form of a first order polynomial in three variables is

$$
\begin{aligned}
c_{0} & +c_{1} v_{1}+c_{2} v_{2}+c_{3} v_{3}+c_{4} v_{1} v_{2} \\
& +c_{5} v_{1} v_{3}+c_{6} v_{2} v_{3}+c_{7} v_{1} v_{2} v_{3},
\end{aligned}
$$


where $c_{i} \in \operatorname{Re}(\operatorname{Re}=$ the real numbers) for $i=0,1, \ldots, 7$. We say that polynomials $P$ and $Q$ are the same if they can both be transformed into the same polynomial $R$ by reordering terms and adding together the coefficients of common products of variables. For example, $P=v_{1} v_{2}+3 v_{1}+4 v_{2}+v_{1} v_{2}$ and $Q=2 v_{1} v_{2}+v_{1}+4 v_{2}+2 v_{1}$ are the same because they can both be transformed to $R=2 v_{1} v_{2}+3 v_{1}+4 v_{2}$. Although for greatest rigor, an equivalence relation among polynomials should be defined by formalizing the notions of reordering terms and summing coefficients of common products, we will assume that this equivalence relation is clear. Equivalence of the polynomials $P$ and $Q$ will be expressed as $P=Q$, since it is the equivalence class of a polynomial and not its typographical form that is of interest here.

First order polynomials can be expressed in a more versatile notation as follows. Let $\mathbf{N}=\{1,2, \ldots, N\}$ and let $W$ be a subset of $\mathbf{N}$. Let $\Pi_{W}$ denote the product of all variables $v_{j}$ for $j \in W$. For example, if $W=\{2,7,9\}$, then $\Pi_{W}=v_{2} v_{7} v_{9}$. We stipulate that $\Pi_{\varnothing}=1$, where $\varnothing$ is the empty set. Under these conventions, the general form of a first order polynomial $P$ in $N$ variables is

$$
P=\sum_{W \subseteq \mathrm{N}} c_{W} \Pi_{W}
$$

where the summation is over the subsets $W$ of $\mathbf{N}$, and each $c_{W}$ is a real number. Every first order polynomial has a unique expression of this form (ignoring changes in the order of addition). Henceforth, we will let FOP abbreviate the expression first order polynomial.

Let $A=A_{1} \times A_{2} \times \cdots \times A_{N}$, where for every $i, A_{i}$ is a nonempty subset of Re. Any $x \in A$ is an ordered sequence, and as a notational convention, $x_{i}$ will denote the $i$ th component of $x$-thus, $x=\left(x_{1}, x_{2}, \ldots, x_{N}\right)$. For each product $\Pi_{W}$ of variables, let $\bar{\Pi}_{w}: A \rightarrow \operatorname{Re}$ be the function taking $x \in A$ to the multiplicative product of the $x_{j}$ for $j \in W$. For example, if $x=(2,5,3,3)$ and $W=\{1,3,4\}$, then $\bar{\Pi}_{W}(x)=2 \cdot 3 \cdot 3=18$. (By convention, $\bar{\Pi}_{\varnothing}(x)=1$ for any $x \in A$ ). We now define the FOP function $\bar{P}: A \rightarrow \operatorname{Re}$ corresponding to $P=\sum c_{W} \Pi_{W}$ by the rule that for any $x \in A$,

$$
\bar{P}(x)=\Sigma c_{w} \bar{\Pi}_{w}(x),
$$

where summation is over all subsets $W$ of $\mathbf{N}$.

The main mathematical result of this section is to identify the following necessary and sufficient condition for a function $F: A \rightarrow \operatorname{Re}$ to be represented by a FOP $P$ in the sense that $\bar{P}=F$. Let $A=A_{1} \times A_{2} \times \cdots \times A_{N} \subseteq \mathrm{Re}^{N}$ for some $N \geqslant 1$. In order to eliminate trivial cases, we assume that every $A_{i}$ contains at least two elements. Let $\bar{A}_{i}$ denote the Cartesian product that is like $A$ except that the $i$ th factor is deleted, i.e.,

$$
\bar{A}_{i}=A_{1} \times \cdots \times A_{i-1} \times A_{i+1} \times \cdots \times A_{N} .
$$

For any $x \in A$, let $\bar{x}_{i}$ denote the element in $\bar{A}_{i}$ that is like $x$ except that the $i$ th component has been deleted, i.e.,

$$
\bar{x}_{i}=\left(x_{1}, \ldots, x_{i-1}, x_{i+1}, \ldots, x_{N}\right) \text {. }
$$


We adopt a convention whereby if $N=1, \bar{A}_{1}=\{\varnothing\}$ and for any $x \in A=A_{1}, \bar{x}_{1}=\varnothing$. The characteristic property of FOP functions is stated in the following definition.

Definition 1. Let $A=A_{1} \times A_{2} \times \cdots \times A_{N} \subseteq \operatorname{Re}^{N}$ and let $F: A \rightarrow \operatorname{Re}$ be any function. We say that $F$ is multiaffine iff for every $i \in \mathbf{N}$ there exist functions $G_{i}: \bar{A}_{i} \rightarrow \operatorname{Re}$ and $H_{i}: \bar{A}_{i} \rightarrow \operatorname{Re}$ such that for every $x \in A$,

$$
F(x)=G_{i}\left(\bar{x}_{i}\right) x_{i}+H_{i}\left(\bar{x}_{i}\right) .
$$

The measurement theory of FOP representations is based on the fact, asserted in Lemma 1, that the multiaffine property is necessary and sufficient for a function $F$ to be represented by a FOP $P$.

LEMMA 1 (Representation lemma for multiaffine functions of real variables). Let $A=A_{1} \times A_{2} \times \cdots \times A_{N}$, where each $A_{i} \subseteq \mathrm{Re}$ contains at least two elements, and let $F: A \rightarrow \operatorname{Re}$ be any function. Then,

(i) There exists a FOP $P$ satisfying $F=\bar{P}$ iff $F$ is multiaffine.

(ii) If $P$ and $Q$ are any FOPs satisfying $\bar{P}=F=\bar{Q}$, then $P=Q$.

It is easy to prove that $F$ is multiaffine whenever $F=\bar{P}$ for some FOP $P$. The converse implication and the uniqueness of $P$ are proved in subsection I(B) by induction on the dimensionality $N$ of $A$.

A minor generalization of Lemma 1 is useful in the context of measurement theory. Let $K=K_{1} \times K_{2} \times \cdots \times K_{N}$ be a Cartesian product of arbitrary sets, and let $F: K \rightarrow \operatorname{Re}$ be any function. We need to define what it means for $F$ to be representable as a FOP.

By a scale $\phi_{i}$ on $K_{i}$, we simply mean any function $\phi_{i}: K_{i} \rightarrow$ Re. Let $\phi=\left(\phi_{1}, \phi_{2}, \ldots, \phi_{N}\right)$ be a sequence of scales on $K_{i}$, for each $i \in \mathbf{N}$. For any $x \in K$, define $\phi(x)$ by

$$
\phi(x)=\left(\phi_{1}\left(x_{1}\right), \phi_{2}\left(x_{2}\right) ; \ldots, \phi_{N}\left(x_{N}\right)\right) .
$$

Suppose $P$ is any FOP in $N$ variables. We let $P \cdot \phi$ denote the polynomial resulting from the replacement of $v_{i}$ by $\phi_{i}$ (for every $i$ ) throughout the polynomial. In other words, let $\Pi_{W}(\phi)$ denote the formal product of scales $\phi_{j}$ for $j \in W$, and then by definition, $P \cdot \phi=\sum c_{W} \Pi_{w}(\phi)$. We regard $\phi_{1}, \phi_{2}, \ldots, \phi_{N}$ as the $N$ variables of the polynomial $P \cdot \phi$. To any $\Pi_{w}(\phi)$ there exists a function $\bar{\Pi}_{w}(\phi): K \rightarrow$ Re defined by $\bar{\Pi}_{w}(\phi)[x]=\bar{\Pi}_{w}(\phi(x))$ for any $x \in K$. In other words, $\bar{\Pi}_{w}(\phi)[x]$ denotes the product of the real numbers $\phi_{i}\left(x_{i}\right)$ for $i \in W$. Corresponding to the polynomial $P \cdot \phi$ is a polynomial function, denoted $\bar{P} \cdot \phi$, that is defined by the condition: for any $x \in K$, $\widetilde{P} \cdot \phi(x)=\sum c_{W} \bar{\Pi}_{w}(\phi)[x]$. It is easy to check that $\bar{P} \cdot \phi(x)=\bar{P}(\phi(x))$. We say that a function $F: K \rightarrow \operatorname{Re}$ is representable by the polynomial $P \cdot \phi$ iff $F=\bar{P} \cdot \phi$.

The distinction between the polynomial $P \cdot \phi$ regarded as a purely formal object, and the function $\bar{P} \cdot \phi: K \rightarrow \operatorname{Re}$ may seem overly technical, but it is useful in 
discussing the uniqueness of representations. For if $\phi$ and $\lambda$ are different sequences of scales, and $P$ and $Q$ are polynomials whose coefficients differ, then necessarily $P \cdot \phi \neq Q \cdot \lambda$, but it is still possible that $\bar{P} \cdot \phi=\bar{Q} \cdot \lambda$. Hence, if $F=\bar{P} \cdot \phi=\bar{Q} \cdot \lambda$, the function $F$ is representable by two different polynomials, $P \cdot \phi$ and $Q \cdot \lambda$.

Definition 1 and Lemma 1 can now be reformulated in a way that is better suited to measurement theoretic endeavors. We maintain the notation whereby

$$
\begin{aligned}
\bar{K}_{i} & =K_{1} \times \cdots \times K_{i-1} \times K_{i+1} \times \cdots \times K_{N}, \\
\bar{x}_{i} & =\left(x_{1}, \ldots, x_{i-1}, x_{i+1}, \ldots, x_{N}\right) .
\end{aligned}
$$

By convention, $\bar{K}_{1}=\{\varnothing\}$ and $\bar{x}_{1}=\varnothing$ when $N=1$. To eliminate trivial cases from the lemma, we assume once again that every $K_{i}$ is essential in the sense that for every $i$, there exist $x, y \in K$ such that $x_{j}=y_{j}$ for all $j \neq i$, and $x_{i} \neq y_{i}$ and $F(x) \neq F(y)$. Some notation will be useful in expressing this more simply. For any $x \in K$ and $a \in K_{i}$, let $\left.x\right|_{i} a$ denote the sequence which is like $x$ except that the $i$ th component has been replaced by $a$, that is,

$$
\left.x\right|_{i} a=\left(x_{1}, \ldots, x_{i-1}, a, x_{i+1}, \ldots, x_{N}\right) .
$$

In these terms, the factor $K_{i}$ is essential iff there exist $a, b \in K_{i}$ and $x \in K$ such that $F\left(\left.x\right|_{i} a\right) \neq F\left(\left.x\right|_{i} b\right)$. To motivate the assumption that every factor is essential, it will be useful to introduce the important concept of a function $F$ that is multiaffine with respect to a sequence $\phi$ of scales.

Definition 2. Let $K=K_{1} \times K_{2} \times \cdots \times K_{N}$ be a Cartesian product of arbitrary sets, let $F: K \rightarrow \operatorname{Re}$ be any function, and let $\phi=\left(\phi_{1}, \phi_{2}, \ldots, \phi_{N}\right)$ be a sequence of scales, where $\phi_{i}: K_{i} \rightarrow \operatorname{Re}$ for every $i \in \mathbf{N}$. Then we say that $F$ is multiaffine with respect to the sequence $\phi$ of scales iff for every $i \in \mathbf{N}$, there exist functions $G_{i}: \bar{K}_{i} \rightarrow \operatorname{Re}$ and $H_{i}: \bar{K}_{i} \rightarrow \operatorname{Re}$ such that for every $x \in K$,

$$
F(x)=G_{i}\left(\bar{x}_{i}\right) \phi_{i}\left(x_{i}\right)+H_{i}\left(\bar{x}_{i}\right) .
$$

When $F$ is multiaffine with respect to $\phi, F\left(\left.x\right|_{i} a\right) \neq F\left(\left.x\right|_{i} b\right)$ implies that $\phi_{i}(a) \neq \phi_{i}(b)$. The main purpose of the assumption that every $K_{i}$ is essential is to establish the existence of $a, b \in K_{i}$ satisfying this last inequality. Using these concepts, Lemma 2 generalizes Lemma 1 to Cartesian products of arbitrary sets.

Lemma 2. Let $K=K_{1} \times K_{2} \times \cdots \times K_{N}$ be a Cartesian product of arbitrary sets and let $F: K \rightarrow \operatorname{Re}$ be any function such that for every $i \in \mathbf{N}$, there exist $a, b \in K_{i}$ and $x \in K$ satisfying $F\left(\left.x\right|_{i} a\right) \neq F\left(\left.x\right|_{i} b\right)$. Then for any sequence $\phi$ of scales,

(i) $F=\bar{P} \cdot \phi$ for some FOP $P$ iff $F$ is multiaffine with respect to $\phi$.

(ii) If $P$ and $Q$ are any FOPs satisfying $\bar{P} \cdot \phi=F=\bar{Q} \cdot \phi$, then $P=Q$.

In addition to the uniqueness result stated in Lemma 2 , we must also consider the 
question of the relationship between FOPs $P$ and $Q$ such that $F=\bar{P} \cdot \phi=\bar{Q} \cdot \lambda$ for distinct sequences $\phi$ and $\lambda$ of scales. To this end, let $F: K \rightarrow \operatorname{Re}$ be any function that is multiaffine with respect to two different sequences $\phi$ and $\lambda$ of scales, and let

$$
P=\sum_{\mathcal{W} \subseteq \mathrm{N}} c_{W} \Pi_{W} \quad \text { and } \quad Q=\sum_{U \subseteq \mathrm{N}}^{\urcorner} d_{U} \Pi_{U}
$$

be two FOPs satisfying $\bar{P} \cdot \phi=F=\bar{Q} \cdot \lambda$. If every factor $K_{i}$ is essential, then for any $i \in \mathbf{N}$, there exist real $\alpha_{i}$ and $\beta_{i}$ such that $\phi_{i}=\alpha_{i} \lambda_{i}+\beta_{i}$ and $\alpha_{i} \neq 0$. Furthermore, letting $\alpha=\left(\alpha_{1}, \alpha_{2}, \ldots, \alpha_{N}\right)$ and $\beta=\left(\beta_{1}, \beta_{2}, \ldots, \beta_{N}\right)$, the coefficients $d_{U}$ of $Q$ are related to the coefficients $c_{W}$ of $P$ by the equation

$$
d_{U}=\bar{\Pi}_{U}(\alpha) \underset{W \supseteq U}{\searrow} c_{W} \bar{\Pi}_{W-U}(\beta)
$$

for any $U \subseteq \mathbf{N}$. Here, $\bar{\Pi}_{U}(\alpha)$ denotes the multiplicative product of the $\alpha_{i}$ for $i \in U$, and $\bar{\Pi}_{W-U}(\beta)$ denotes the multiplicative product of the $\beta_{j}$ for $j \in W-U$. Since $\bar{\Pi}_{\varnothing}(\alpha)=\Pi_{\varnothing}(\beta)=1$ by convention, the preceding expression can be simplified in the case where $U=\varnothing$ to

$$
d_{\varnothing}=\sum_{W \subseteq \mathrm{N}} c_{W} \bar{\Pi}_{W}(\beta) .
$$

As Lemma 3 asserts, these relationships between $\phi$ and $\lambda$ and between the coefficients of $P$ and $Q$ are sufficient as well as necessary for $\bar{P} \cdot \phi=\bar{Q} \cdot \lambda$.

Lemma 3. Let $K_{1} \times K_{2} \times \cdots \times K_{N}$ be a Cartesian product of arbitrary sets, and let $F: K \rightarrow \operatorname{Re}$ be any function such that for every $i \in \mathbf{N}$, there exist $a, b \in K_{i}$ and $x \in K$ satisfying $F\left(\left.x\right|_{i} a\right) \neq F\left(\left.x\right|_{i} b\right)$. Suppose $P$ is $a$ FOP and $\phi$ a sequence of scales such that $F=\bar{P} \cdot \phi$, and let $Q$ be any other FOP and $\lambda$ any other sequence of scales. If we express $P$ and $Q$ in the form

$$
P=\sum_{W \subseteq \mathrm{N}} c_{W} \Pi_{W} \quad \text { and } \quad Q=\sum_{U \subseteq \mathrm{N}} d_{U} \Pi_{U},
$$

then $\bar{P} \cdot \phi=F=\bar{Q} \cdot \lambda$ iff (i) and (ii) hold:

(i) For every $i \in \mathbf{N}$, there exist $\alpha_{i}, \beta_{i} \in \operatorname{Re}$ such that $\phi_{i}=\alpha_{i} \lambda_{i}+\beta_{i}$ and $\alpha_{i} \neq 0$. Then

(ii) Let $\alpha=\left(\alpha_{1}, \alpha_{2}, \ldots, \alpha_{N}\right)$ and $\beta=\left(\beta_{1}, \beta_{2}, \ldots, \beta_{N}\right)$ for the $\alpha_{i}$ and $\beta_{i}$ satisfying (i).

$$
d_{U}=\bar{\Pi}_{U}(\alpha) \underset{W \supseteq U}{\sum_{W}} c_{W} \bar{\Pi}_{W-U}(\beta)
$$

for any $U \subseteq \mathbf{N}$, and

$$
d_{\varnothing}=\sum_{W \subseteq \mathrm{N}} c_{W} \bar{\Pi}_{W}(\beta)
$$




\section{I(B). Proofs}

Proof of Lemma 1. First, we prove that if there exists a FOP $P$ satisfying $F=\bar{P}$, then $F$ is multiaffine. Suppose that $F=\bar{P}$ for some FOP $P$. Let $\mathbf{N}^{*}$ denote the power set of $\mathbf{N}$. For any $i$, partition $\mathbf{N}^{*}$ into subsets $\Gamma_{i}$ and $\Delta_{i}$ by the conditions $w \in \Gamma_{i}$ iff $i \in W$ and $W \in \Delta_{i}$ iff $i \notin W$. Now

$$
\begin{aligned}
P & =\sum_{W \in \mathbb{N}^{*}} c_{W} \Pi_{W}=\sum_{W \in \Gamma_{i}} c_{W} \Pi_{W}+\sum_{W \in \Delta_{i}} c_{W} \Pi_{W} \\
& =\left(\sum_{W \in \Gamma_{i}} c_{W} \Pi_{W-(i)}\right) v_{i}+\sum_{W \in \Delta_{i}} c_{W} \Pi_{W} .
\end{aligned}
$$

Let $R_{i}=\sum_{W \in \Gamma_{i}} c_{W} \Pi_{W-(i)}$ and $S_{i}=\sum_{W \in \Delta_{i}} c_{W} \Pi_{W}$. Then $P=R_{i} v_{i}+S_{i}$, so

$$
F(x)=\bar{P}(x)=\bar{R}_{i}\left(\bar{x}_{i}\right) x_{i}+\bar{S}_{i}\left(\bar{x}_{i}\right)
$$

for any $x \in A$. Hence $F$ is multiaffine. This proves half of part (i) of the lemma.

Note that if the hypotheses of part (ii) are satisfied, i.e., that $P$ and $Q$ are FOPs satisfying $\bar{P}=F=\bar{Q}$, then by what we have just proved, $F$ is multiaffine. If we now show that to any multiaffine function $F$ there exists a unique FOP $P$ satisfying $F=\bar{P}$, then the remaining half of part (i) and part (ii) of the lemma will be established. Let $F$ be a multiaffine function. We prove that there exists a unique FOP $P$ (up to equivalence) satisfying $F=\bar{P}$ by induction on $N$, the dimensionality of the domain of $F$.

For $N=1, A=A_{1}$ and $F$ multiaffine means that there exist $G_{1}$ and $H_{1}$ satisfying

$$
F(x)=G_{1}(\varnothing) x+H_{1}(\varnothing)
$$

for every $x \in A$. Let $P=G_{1}(\varnothing) v_{1}+H_{1}(\varnothing)$. Evidently, $F(x)=\bar{P}(x)$ for any $x \in A$. Let $Q=\alpha v_{1}+\beta$ be any other FOP of one variable satisfying $F=\bar{Q}$. Choose $x, y \in A$ such that $F(x) \neq F(y)$. Since $\bar{P}=F=\bar{Q}$,

$$
\begin{aligned}
& G_{1}(\varnothing) x+H_{1}(\varnothing)=\alpha x+\beta, \\
& G_{1}(\varnothing) y+H_{1}(\varnothing)=\alpha y+\beta .
\end{aligned}
$$

Therefore $G_{1}(\varnothing)(x-y)=\alpha(x-y)$. Since $x-y \neq 0, G_{1}(\varnothing)=\alpha$. Hence, $H_{1}(\varnothing)=\beta$. Hence $P=Q$, and the lemma holds for $N=1$.

Now assume the lemma holds for some $N \geqslant 1$, and we establish it for $N+1$. For notational simplicity, let $M$ denote $N+1$. Let $F$ be a multiaffine function with domain $A=A_{1} \times \cdots \times A_{M}$. For any $t \in A_{M}$, define a function $F_{t}: \bar{A}_{M} \rightarrow \operatorname{Re}$ as follows. For any $u \in \bar{A}_{M}$, choose $x \in A$ such that $\bar{x}_{M}=u$ and let $F_{t}(u)=F\left(\left.x\right|_{M} t\right)$, where the notation $\left.x\right|_{M} t$ is defined in the text. Note that the value of $F_{t}(u)$ does not depend on the particular $x$ chosen, for if $z \in A$ is any other element satisfying $\bar{z}_{M}=u$, then $\left.z\right|_{M} t=\left.x\right|_{M} t$. By our notational conventions, $\left(\overline{\left.x\right|_{M} t}\right)_{k}$ denotes the sequence that is 
like $\left.x\right|_{M} t$ except that the $k$ th component is omitted. Since $F$ is multiaffine by hypothesis, we have for any $x \in A$ and any $k \in \mathbf{N}$, that

$$
\left.F_{t}\left(\bar{x}_{M}\right)=F\left(\left.x\right|_{M} t\right)=G_{k}\left(\left(\overline{\left.x\right|_{M} t}\right)_{k}\right) x_{k}+H_{k}\left(\overline{\left(\overline{\left.x\right|_{M}} t\right.}\right)_{k}\right) .
$$

For any $k \in \mathbf{N}$, let $\bar{A}_{k M}$ denote the Cartesian product that is like $\bar{A}_{M}$ except that the factor $A_{k}$ has been deleted, and let $\bar{x}_{k M}$ denote the element of $\bar{A}_{k M}$ that is like $x \in A$ except that the $k$ th and the $M$ th components have been omitted. $\left(\bar{A}_{k M}=\{\varnothing\}\right.$ and $\bar{x}_{k M}=\varnothing$ when $M=2$ ). Define functions $G_{k}^{*}: \bar{A}_{k M} \rightarrow \operatorname{Re}$ and $H_{k}^{*}: \bar{A}_{k M} \rightarrow \operatorname{Re}$ by

$$
G_{k}^{*}\left(\bar{x}_{k M}\right)=G_{k}\left(\left(\overline{\left.x\right|_{M} t}\right)_{k}\right), \quad H_{k}^{*}\left(\bar{x}_{k M}\right)=H_{k}\left(\left(\overline{\left.x\right|_{M} t}\right)_{k}\right) .
$$

These function are well defined because if $\bar{x}_{k M}=\bar{y}_{k M}$, then $\left.x\right|_{M} t$ and $\left.y\right|_{M} t$ differ at most on the $k$ th component, so $\left(\overline{\left.x\right|_{M} t}\right)_{k}=\left(\overline{\left.y\right|_{M} t}\right)_{k}$.

But now for any $u \in \bar{A}_{M}$ and $k \in \mathbf{N}$, choose $x \in A$ such that $\bar{x}_{M}=u$, and (2) can be rewritten as

$$
\begin{aligned}
F_{t}(u) & =F\left(\left.x\right|_{M} t\right)=G_{k}^{*}\left(\bar{x}_{k M}\right) x_{k}+H_{k}^{*}\left(\bar{x}_{k M}\right) \\
& =G_{k}^{*}\left(\bar{u}_{k}\right) u_{k}+H_{k}^{*}\left(\bar{u}_{k}\right)
\end{aligned}
$$

since $u_{k}=x_{k}$ and $\bar{u}_{k}=\bar{x}_{k M}$. Thus, $F_{t}$ is a multiaffine function whose domain $\bar{A}_{M}$ has $N$ factors $A_{1}, \ldots, A_{N}$. By the induction hypothesis there exists a FOP $Q_{t}$ in the variables $v_{1}, \ldots, v_{N}$ such that $F_{t}=\bar{Q}_{t}$. Since $t \in A_{M}$ was chosen arbitrarily, any $t \in A_{M}$ determines a function $F_{t}: \bar{A}_{M} \rightarrow \operatorname{Re}$ and a FOP $Q_{t}$ satisfying $F_{t}=\bar{Q}_{t}$.

Note that for any $x \in A$ and $t \in A_{M}$, we have $\left(\overline{x||_{M} t}\right)_{M}=\bar{x}_{M}$ by the definitions of the operations $\left.\right|_{M}$ and $\left({ }^{-}\right)_{M}$. Since $F$ is multiaffine, we have

$$
\begin{aligned}
F_{t}\left(\bar{x}_{M}\right) & =F\left(\left.x\right|_{m} t\right) \\
& \left.=G_{M}\left(\left(\overline{\left.x\right|_{M} t}\right)_{M}\right) t+H_{M}\left(\overline{\left(\left.x\right|_{M} t\right.}\right)_{M}\right) \\
& =G_{M}\left(\bar{x}_{M}\right) t+H_{M}\left(\bar{x}_{M}\right) .
\end{aligned}
$$

A similar derivation yields $F_{s}\left(\bar{x}_{M}\right)=G_{M}\left(\bar{x}_{M}\right) s+H_{M}\left(\bar{x}_{M}\right)$ for any other $s \neq t$ such that $s \in A_{M}$. Hence

$$
F_{t}\left(\bar{x}_{M}\right)-F_{s}\left(\bar{x}_{M}\right)=G_{M}\left(\bar{x}_{M}\right)(t-s) .
$$

Let $Q_{t}$ and $Q_{s}$ be FOPs satisfying $\bar{Q}_{t}=F_{t}$ and $\bar{Q}_{s}=F_{s}$, for any fixed choice of $t, s \in A_{M}$ such that $t \neq s$. Define the polynomial $R$ by $R=(t-s)^{-1}\left(Q_{t}-Q_{s}\right)$. Since $Q_{t}$ and $Q_{s}$ are FOPs in the variables $v_{1}, \ldots, v_{N}, R$ is also a FOP in these variables. Moreover, (4) implies that $G_{M}\left(\bar{x}_{M}\right)=\vec{R}\left(\bar{x}_{M}\right)$ for any $x \in A$. From (3) and the identities $\bar{Q}_{t}=F_{t}$ and $\bar{R}=G_{M}$, we have $H_{M}\left(\bar{x}_{M}\right)=\bar{Q}_{t}\left(\bar{x}_{M}\right)-\bar{R}\left(\bar{x}_{M}\right) t$. Let $S$ be the polynomial determined by $S=Q_{t}-t R$. Evidently $S$ is a FOP in the variables 
$v_{1}, \ldots, v_{N}$ satisfying $H_{M}=\bar{S}$. Define a polynomial $P$ by $P=R v_{M}+S$. Since $v_{M}$ is not a variable of $R, R v_{M}$ is a FOP, hence so is $P$. For any $x \in A$,

$$
\begin{aligned}
F(x) & =G_{M}\left(\bar{x}_{M}\right) x_{M}+H_{M}\left(\bar{x}_{M}\right) \\
& =\bar{R}\left(\bar{x}_{M}\right) x_{M}+\bar{S}\left(\bar{x}_{M}\right)=\overline{R v_{M}+S}(x) \\
& =\bar{P}(x) .
\end{aligned}
$$

Thus $F=\bar{P}$ for the FOP $P$.

Now let $Q=\sum d_{U} \Pi_{U}$ be any other FOP satisfying $F=\bar{Q}$. Letting $\Gamma_{i}$ and $\Delta_{i}$ be the partition of $\mathbf{M}^{*}$, the power set of $\mathbf{M}$, defined at the beginning of the proof, we must have

$$
\begin{aligned}
Q & =\sum_{U \in \mathrm{M}^{*}} d_{U} \Pi_{U}=\sum_{U \in \Gamma_{M}} d_{U} \Pi_{U}+\sum_{U \in \Delta_{M}} d_{U} \Pi_{U} \\
& =\left(\sum_{U \in \Gamma_{M}} d_{U} \Pi_{U-(M)}\right) v_{M}+\sum_{U \in \Delta_{M}} d_{U} \Pi_{U} \\
& =R^{\prime} v_{M}+S^{\prime},
\end{aligned}
$$

where $R^{\prime}=\sum d_{V} \Pi_{U-\{M\}}$ and $S^{\prime}=\sum d_{U} \Pi_{U}$ (summation over elements of $\Gamma_{M}$ and $\Delta_{M}$ ). Note that $R^{\prime}$ and $S^{\prime}$ are FOPs in the $N$ variables $v_{1}, \ldots, v_{N}$.

Since $\bar{P}=\bar{Q}$, we have

$$
\bar{R}\left(\bar{x}_{M}\right) x_{M}+\bar{S}\left(\bar{x}_{M}\right)=\bar{R}^{\prime}\left(\bar{x}_{M}\right) x_{M}+\bar{S}^{\prime}\left(\bar{x}_{M}\right)
$$

for any $x \in A$. Successively setting $x_{M}$ equal to elements $a$ and $b$ of $A_{M}$ such that $a \neq b$ yields

$$
\begin{aligned}
& \bar{R}\left(\bar{x}_{M}\right) a+\bar{S}\left(\bar{x}_{M}\right)=\bar{R}^{\prime}\left(\bar{x}_{M}\right) a+\bar{S}^{\prime}\left(\bar{x}_{M}\right), \\
& \bar{R}\left(\bar{x}_{M}\right) b+\bar{S}\left(\bar{x}_{M}\right)=\bar{R}^{\prime}\left(\bar{x}_{M}\right) b+\bar{S}^{\prime}\left(\bar{x}_{M}\right) .
\end{aligned}
$$

Hence $\quad \bar{R}\left(\bar{x}_{M}\right)(a-b)=\bar{R}^{\prime}\left(\bar{x}_{M}\right)(a-b)$. Since $a-b \neq 0$, we must have $\bar{R}\left(\bar{x}_{M}\right)=\bar{R}^{\prime}\left(\bar{x}_{M}\right)$, and furthermore, this implies $\bar{S}\left(\bar{x}_{M}\right)=\bar{S}^{\prime}\left(\bar{x}_{M}\right)$. Since $x \in A$ was arbitrary, we have proved $\bar{R}=\bar{R}^{\prime}$ and $\bar{S}=\bar{S}^{\prime}$. The domain $\bar{A}_{M}$ of $\bar{R}$ and $\bar{S}$ has $N$ factors, and hence, the induction hypothesis implies that $R=R^{\prime}$ and $S=S^{\prime}$. Therefore, $P=R v_{M}+S=Q$.

Q.E.D.

Proof of Lemma 2. We first prove part (i) of Lemma 2 for the case $N=1$. Suppose $N=1$ and $F=\bar{P} \cdot \phi$ for some FOP $P$. Then $P \cdot \phi=\alpha \phi_{1}+\beta$ for real $\alpha$ and $\beta$. Define $G_{1}$ and $H_{1}$ by $G_{1}(\varnothing)=\alpha$ and $H_{1}(\varnothing)=\beta$. Then $F(x)=G_{1}\left(\bar{x}_{1}\right) \phi_{1}\left(x_{1}\right)+H_{1}\left(\bar{x}_{1}\right)$ for any $x \in K=K_{1}$ so $F$ is multiaffine with respect to $\phi$. Conversely, if $F$ is multiaffine with respect to $\phi=\left(\phi_{1}\right)$, then $P=G_{1}(\varnothing) v_{1}+H_{1}(\varnothing)$ satisfies $F=\bar{P} \cdot \phi$.

Now suppose that $N>1$. We prove directly that $F=\bar{P} \cdot \phi$ for some FOP $P$ iff $F$ is multiaffine with respect to $\phi$. First suppose that $F=\bar{P} \cdot \phi$ for some FOP $P$. From the proof of Lemma 1, we know that for any $i \in \mathbf{N}$, there exist FOPs $R_{i}$ and $S_{i}$ in the 
variables $v_{j}$ for $j \neq i$ such that $P=R_{i} v_{i}+S_{i}$. Let $\bar{\phi}_{i}$ denote the sequence of scales that is like $\phi$ but with the $i$ th component omitted. Then for any $x \in K$,

$$
\begin{aligned}
F(x) & =\bar{P} \cdot \phi(x)=\bar{P}(\phi(x)) \\
& =\bar{R}_{i}\left(\bar{\phi}_{i}\left(\bar{x}_{i}\right)\right) \phi_{i}\left(x_{i}\right)+\bar{S}_{i}\left(\bar{\phi}_{i}\left(\bar{x}_{i}\right)\right) .
\end{aligned}
$$

Hence $F$ is multiaffine with respect to $\phi$.

Next suppose that $F$ is multiaffine with respect to $\phi$. Let $\operatorname{Im} \phi$ denote the set of all $\eta \in \operatorname{Re}^{N}$ such that $\eta=\phi(x)$ for some $x \in K$, and define $\operatorname{Im} \bar{\phi}_{i}$ similarly (for every $i \in \mathbf{N}$ ). Suppose there exists a function $F^{*}: \operatorname{Im} \phi \rightarrow \operatorname{Re}$, and for every $i$, there exist $a, b \in K_{i}$ and functions $G_{i}^{*}: \operatorname{Im} \bar{\phi}_{i} \rightarrow \operatorname{Re}$ and $H_{i}^{*}: \operatorname{Im} \bar{\phi}_{i} \rightarrow \operatorname{Re}$ such that

$$
\begin{aligned}
F^{*}(\phi(x)) & =F(x), \\
\phi_{i}(a) & \neq \phi_{i}(b), \\
G_{i}^{*}\left(\bar{\phi}_{i}\left(\bar{x}_{i}\right)\right) & =G_{i}\left(\bar{x}_{i}\right), \\
H_{i}^{*}\left(\bar{\phi}_{i}\left(\bar{x}_{i}\right)\right) & =H_{i}\left(\bar{x}_{i}\right),
\end{aligned}
$$

for all $x \in K$. We first show that these assumptions yield an easy proof of the existence and uniqueness of the FOP $P$ satisfying $F=\bar{P} \cdot \phi$, and then show that if $F$ is multiaffine with respect to $\phi$, the desired functions $F^{*}, G_{i}^{*}$, and $H_{i}^{*}$, and elements $a, b \in K_{i}$ exist.

Since $F$ is multiaffine with respect to $\phi$, and assuming the existence of $F^{*}, G_{i}^{*}$, and $H_{i}^{*}$, we must have

$$
\begin{aligned}
F^{*}(\phi(x)) & =F(x) \\
& =G_{i}\left(\bar{x}_{i}\right) \phi_{i}\left(x_{i}\right)+H_{i}\left(\bar{x}_{i}\right) \\
& =G_{i}^{*}\left(\bar{\phi}_{i}\left(\bar{x}_{i}\right)\right) \phi_{i}\left(x_{i}\right)+H_{i}^{*}\left(\bar{\phi}_{i}\left(\bar{x}_{i}\right)\right)
\end{aligned}
$$

for any $\phi(x) \in \operatorname{Im} \phi$ and $i \in \mathbf{N}$. Hence $F^{*}$ is multiaffine in the sense of definition 1. Since

$$
\operatorname{Im} \phi=\phi_{1}\left(K_{1}\right) \times \phi_{2}\left(K_{2}\right) \times \cdots \times \phi_{N}\left(K_{N}\right)
$$

and we are assuming that for every $i$, there exist $a, b \in K_{i}$ such that $\phi_{i}(a) \neq \phi_{i}(b)$, the hypotheses of Lemma 1 are satisfied and there exists a FOP $P$ such that $F^{*}=\bar{P}$. Therefore $F=F^{*} \cdot \phi=\bar{P} \cdot \phi$ and part (i) of the lemma has been proven. To prove part (ii), let $Q$ be any other FOP satisfying $F=\bar{Q} \cdot \phi$. Since this implies that $\bar{P}=F^{*}=\bar{Q}$, we may invoke Lemma 1 to deduce $P=Q$. Thus the proof of Lemma 2 is completed once the existence of the required functions $F^{*}, G_{i}^{*}, H_{i}^{*}$ and elements $a, b \in K_{i}$ has been established under the assumption that $F$ is multiaffine with respect to $\phi$.

We first show that $\phi(x)=\phi(y)$ implies that $F(x)=F(y)$ for any $x, y \in K$. Suppose $\phi(x)=\phi(y)$ and define a sequence $u^{0}, u^{1}, \ldots, u^{N}$ of elements of $K$ by 


$$
\begin{array}{ccc}
u^{0}= & \left(x_{1}, x_{2}, \ldots, x_{N}\right) & =x, \\
u^{1}= & \left(y_{1}, x_{2}, \ldots, x_{N}\right) & =\left.u^{0}\right|_{1} y_{1}, \\
\vdots & & \vdots \\
u^{k}= & \left(y_{1}, \ldots, y_{k}, x_{k+1}, \ldots, x_{N}\right)=\left.u^{k-1}\right|_{k} y_{k}, \\
\vdots & & \vdots \\
u^{N}= & \left(y_{1}, y_{2}, \ldots, y_{N}\right) & =\left.u^{N-1}\right|_{N} y_{N}=y .
\end{array}
$$

We show by induction that $F(x)=F\left(u^{j}\right)$ for all $j \in \mathbf{N}$. If $j=0, F(x)=F\left(u^{0}\right)$ by definition of $u^{0}$. Now suppose $F(x)=F\left(u^{j}\right)$ for all $j \leqslant k<N$, and we prove $F(x)=F\left(u^{k+1}\right)$. For notational simplicity, let $m=k+1$, let $v=u^{k}$ and let $w=u^{k+1}$. The sequences $v$ and $w$ differ only on the $m$ th component since $w=\left.v\right|_{m} y_{m}$ by definition. Hence $\bar{v}_{m}=\bar{w}_{m}$. Moreover, $\phi_{m}\left(v_{m}\right)=\phi_{m}\left(x_{m}\right)=\phi_{m}\left(y_{m}\right)=\phi_{m}\left(w_{m}\right)$ because $\phi(x)=\phi(y)$. Hence, these identities and the fact that $F$ is multiaffine with respect to $\phi$ yields that

$$
\begin{aligned}
F(x) & =F(v)=G_{m}\left(\bar{v}_{m}\right) \phi_{m}\left(v_{m}\right)+H_{m}\left(\bar{v}_{m}\right) \\
& =G_{m}\left(\bar{w}_{m}\right) \phi_{m}\left(w_{m}\right)+H_{m}\left(\bar{w}_{m}\right) \\
& =F(w)=F\left(u^{k+1}\right) .
\end{aligned}
$$

Hence, $F(x)=F\left(u^{N}\right)=F(y)$, and we have proved that $\phi(x)=\phi(y)$ implies $F(x)=F(y)$. But this shows that the condition $F^{*}(\phi(x))=F(x)$ determines a welldefined function $F^{*}: \operatorname{Im} \phi \rightarrow \operatorname{Re}$, so the existence of $F^{*}$ has been established.

Now we show that for any $i \in \mathbf{N}$, there exist $a, b \in K_{i}$ such that $\phi_{i}(a) \neq \phi_{i}(b)$. Note that for any $x \in K$ and $a \in K_{i},\left(\overline{\left.x\right|_{i} a}\right)_{i}=\bar{x}_{i}$ by definition of the operations $\left({ }^{-}\right)_{i}$ and $l_{i}$. Now choose $x \in K$ and $a, b \in K_{i}$ such that $F\left(\left.x\right|_{i} a\right) \neq F\left(\left.x\right|_{i} b\right)$. Since $F$ is multiaffine with respect to $\phi$,

$$
\begin{aligned}
G_{i}\left(\bar{x}_{i}\right) \phi_{i}(a)+H_{i}\left(\bar{x}_{i}\right) & \left.=G_{i}\left(\left(\overline{\left.x\right|_{i} a}\right)_{i}\right) \phi_{i}(a)+H_{i}\left(\overline{\left(\overline{\left.x\right|_{i} a}\right.}\right)_{i}\right) \\
& =F\left(\left.x\right|_{i} a\right) \\
& \neq F\left(\left.x\right|_{i} b\right) \\
& \left.=G_{i}\left(\left(\overline{\left.x\right|_{i} b}\right)_{i}\right) \phi_{i}(b)+H_{i}\left(\overline{\left(\left.x\right|_{i} b\right.}\right)_{i}\right) \\
& =G_{i}\left(\bar{x}_{i}\right) \phi_{i}(b)+H_{i}\left(\bar{x}_{i}\right) .
\end{aligned}
$$

Therefore $\phi_{i}(a) \neq \phi_{i}(b)$.

Finally we show that $\bar{\phi}_{i}\left(\bar{x}_{i}\right)=\bar{\phi}_{i}\left(\bar{y}_{i}\right)$ implies that $G_{i}\left(\bar{x}_{i}\right)=G_{i}\left(\bar{y}_{i}\right)$ and $H_{i}\left(\bar{x}_{i}\right)=H_{i}\left(\bar{y}_{i}\right)$ for any $x, y \in K$. Suppose $\bar{\phi}_{i}\left(\bar{x}_{i}\right)=\bar{\phi}_{i}\left(\bar{y}_{i}\right)$. Then $\phi\left(\left.x\right|_{i} t\right)=\phi\left(\left.y\right|_{i} t\right)$ for any $t \in K_{i}$, and hence $F\left(\left.x\right|_{i} t\right)=F\left(\left.y\right|_{i} t\right)$. But then

$$
\begin{aligned}
G_{i}\left(\bar{x}_{i}\right) \phi_{i}(t)+H_{i}\left(\bar{x}_{i}\right) & =F\left(\left.x\right|_{i} t\right)=F\left(\left.y\right|_{i} t\right) \\
& =G_{i}\left(\bar{y}_{i}\right) \phi_{i}(t)+H_{i}\left(\bar{y}_{i}\right) .
\end{aligned}
$$


Hence $\left[G_{i}\left(\bar{x}_{i}\right)-G_{i}\left(\bar{y}_{i}\right)\right] \phi_{i}(t)+\left[H_{i}\left(\bar{x}_{i}\right)-H_{i}\left(\bar{y}_{i}\right)\right]=0$. Since this last relationship holds when we set $t$ equal to elements $a$ or $b$ of $K_{i}$ such that $\phi_{i}(a) \neq \phi_{i}(b)$, we must have $G_{i}\left(\bar{x}_{i}\right)=G_{i}\left(\bar{y}_{i}\right)$ and $H_{i}\left(\bar{x}_{i}\right)=H_{i}\left(\bar{y}_{i}\right)$. Having established that $\bar{\phi}_{i}\left(\bar{x}_{i}\right)=\bar{\phi}_{i}\left(\bar{y}_{i}\right)$ implies $G_{i}\left(\bar{x}_{i}\right)=G_{i}\left(\bar{y}_{i}\right)$ and $H_{i}\left(\bar{x}_{i}\right)=H_{i}\left(\bar{y}_{i}\right)$, it is clear that the conditions $G_{i}^{*}\left(\bar{\phi}_{i}\left(\bar{x}_{i}\right)\right)=G_{i}\left(\bar{x}_{i}\right)$ and $H_{i}^{*}\left(\bar{\phi}_{i}\left(\bar{x}_{i}\right)\right)=H_{i}\left(\bar{x}_{i}\right)$ determine well defined functions $G_{i}^{*}: \operatorname{Im} \bar{\phi}_{i} \rightarrow \operatorname{Re}$ and $H_{i}^{*}: \operatorname{Im} \bar{\phi}_{i} \rightarrow \operatorname{Re}$.

Q.E.D.

Proof of Lemma 3. First assume that $\bar{P} \cdot \phi=F=\bar{Q} \cdot \lambda$. We show that (i) and (ii) hold.

(i) Since Lemma 2 implies that $F$ is multiaffine with respect to $\phi$ and $\lambda$, there exist functions $G_{i}, H_{i}, g_{i}$, and $h_{i}$ satisfying

$$
\begin{aligned}
F(x) & =G_{i}\left(\bar{x}_{i}\right) \phi_{i}\left(x_{i}\right)+H_{i}\left(\bar{x}_{i}\right) \\
& =g_{i}\left(\bar{x}_{i}\right) \lambda_{i}\left(x_{i}\right)+h_{i}\left(\bar{x}_{i}\right)
\end{aligned}
$$

for any $i \in \mathbf{N}$ and $x \in K$. Now choose $u \in K$ and $a, b \in K_{i}$ such that $F\left(\left.u\right|_{i} a\right) \neq F\left(\left.u\right|_{i} b\right)$. Since $\left(\overline{\left.x\right|_{i} t}\right)_{i}=\bar{x}_{i}$ for any $x \in K$ and $t \in K_{i}$, we must have

$$
\begin{aligned}
0 \neq F\left(\left.u\right|_{i} a\right)-F\left(\left.u\right|_{i} b\right) & =G_{i}\left(\bar{u}_{i}\right)\left[\phi_{i}(a)-\phi_{i}(b)\right] \\
& =g_{i}\left(\bar{u}_{i}\right)\left[\lambda_{i}(a)-\lambda_{i}(b)\right] .
\end{aligned}
$$

Hence $G_{i}\left(\bar{u}_{i}\right) \neq 0, g_{i}\left(\bar{u}_{i}\right) \neq 0, \phi_{i}(a) \neq \phi_{i}(b)$, and $\lambda_{i}(a) \neq \lambda_{i}(b)$. Furthermore,

$$
\begin{aligned}
F\left(\left.u\right|_{i} t\right) & =G_{i}\left(\bar{u}_{i}\right) \phi_{i}(t)+H_{i}\left(\bar{u}_{i}\right) \\
& =g_{i}\left(\bar{u}_{i}\right) \lambda_{i}(t)+h_{i}\left(\bar{u}_{i}\right)
\end{aligned}
$$

for any $t \in K_{i}$. Therefore

$$
\begin{aligned}
\phi_{i}(t) & =\left[g_{i}\left(\bar{u}_{i}\right) / G_{i}\left(\bar{u}_{i}\right)\right] \lambda_{i}(t)+\left[h_{i}\left(\bar{u}_{i}\right)-H_{i}\left(\bar{u}_{i}\right)\right] / G_{i}\left(\bar{u}_{i}\right) \\
& =\alpha_{i}\left(\bar{u}_{i}\right) \lambda_{i}(t)+\beta_{i}\left(\bar{u}_{i}\right),
\end{aligned}
$$

where $\alpha_{i}\left(\bar{u}_{i}\right)=\left[g_{i}\left(\bar{u}_{i}\right) / G_{i}\left(\bar{u}_{i}\right)\right] \neq 0$ and $\beta_{i}\left(\bar{u}_{i}\right)=\left[h_{i}\left(\bar{u}_{i}\right)-H_{i}\left(\bar{u}_{i}\right)\right] / G_{i}\left(\bar{u}_{i}\right)$. To see that $\alpha_{i}\left(\bar{u}_{i}\right)$ and $\beta_{i}\left(\bar{u}_{i}\right)$ do not really depend on $\bar{u}_{i}$, suppose that $\phi_{i}(t)=\alpha_{i}\left(\bar{w}_{i}\right) \lambda_{i}(t)+\beta_{i}\left(\bar{w}_{i}\right)$ for some other $\bar{w}_{l}$. Then $\left[\alpha_{i}\left(\bar{u}_{i}\right)-\alpha_{i}\left(\bar{w}_{i}\right)\right] \lambda_{i}(t)=\beta_{i}\left(\bar{w}_{i}\right)-\beta_{i}\left(\bar{u}_{i}\right)$. Since there exists $a, b \in K_{i}$ such that $\lambda_{i}(a) \neq \lambda_{i}(b)$, it must be that $\alpha_{i}\left(\bar{u}_{i}\right)-\alpha_{i}\left(\bar{w}_{i}\right)=0$ and $\beta_{i}\left(\bar{u}_{i}\right)-$ $\beta_{i}\left(\bar{w}_{i}\right)=0$. Hence, $\phi_{i}(t)=\alpha_{i} \lambda_{i}(t)+\beta_{i}$ for $\alpha_{i}=\alpha_{i}\left(\bar{u}_{i}\right)$ and $\beta_{i}=\beta_{i}\left(\bar{u}_{i}\right)$ for any choice of $\bar{u}_{i}$ such that $G_{i}\left(\bar{u}_{i}\right) \neq 0$.

(ii) Let $\alpha=\left(\alpha_{1}, \alpha_{2}, \ldots, \alpha_{N}\right)$ and $\beta=\left(\beta_{1}, \beta_{2}, \ldots, \beta_{N}\right)$ and define $\alpha \lambda$ and $\alpha \lambda+\beta$ to be the sequences

$$
\begin{aligned}
a \lambda & =\left(\alpha_{1} \lambda_{1}, \alpha_{2} \lambda_{2}, \ldots, \alpha_{N} \lambda_{N}\right), \\
a \lambda+\beta & =\left(\alpha_{1} \lambda_{1}+\beta_{1}, \alpha_{2} \lambda_{2}+\beta_{2}, \ldots, \alpha_{N} \lambda_{N}+\beta_{N}\right) .
\end{aligned}
$$


By part (i), $\alpha \lambda+\beta$ and $\phi$ are identical sequences of scales. Therefore $\bar{\Pi}_{W}(\phi)=\bar{\Pi}_{W}(\alpha \lambda+\beta)$ for any $W \subseteq N$. But a simple induction on the size of the set $W$ gives

$$
\bar{\Pi}_{W}(\alpha \lambda+\beta)=\sum_{U \subseteq w} \bar{\Pi}_{U}(\alpha \lambda) \bar{\Pi}_{W-U}(\beta)
$$

Hence

$$
\begin{aligned}
\bar{P} \cdot \phi & =\sum_{W \subseteq \mathrm{N}} c_{W} \bar{\Pi}_{W}(\phi)=\sum_{W \subset \mathrm{N}} c_{W} \bar{\Pi}_{W}(\alpha \lambda+\beta) \\
& =\sum_{W \subseteq \mathrm{N}} c_{W}\left[\sum_{U \subseteq W} \Pi_{U}(\alpha \lambda) \bar{\Pi}_{W-U}(\beta)\right] \\
& =\sum_{U \subseteq \mathrm{N}} \bar{\Pi}_{U}(\alpha \lambda)\left[\sum_{W \supseteq U} c_{W} \bar{\Pi}_{W-U}(\beta)\right] \\
& =\sum_{U \subseteq \mathrm{N}}\left[\bar{\Pi}_{U}(\alpha) \underset{W \supseteq U}{\sum_{W}} c_{W} \bar{\Pi}_{W-U}(\beta)\right] \bar{\Pi}_{U}(\lambda) .
\end{aligned}
$$

Let $Q^{\prime}=\sum e_{V I} \Pi_{V t}$ be the FOP whose coefficients are determined by the condition

$$
e_{U}=\bar{\Pi}_{U}(\alpha) \sum_{W \supseteq U} c_{W} \bar{\Pi}_{W-U}(\beta)
$$

Then (5) implies $\bar{Q}^{\prime} \cdot \lambda=\bar{P} \cdot \phi=\bar{Q} \cdot \lambda$. But now, the hypotheses of Lemma 2(ii) are satisfied by $\bar{Q}^{\prime} \cdot \lambda$ and $\bar{Q} \cdot \lambda$, so $Q^{\prime}=Q$. Hence $d_{U}=e_{U}$ for every $U \subseteq \mathbf{N}$, so the expression for $d_{U}$ in part (ii) is established. Evidently, the expression for $d_{U}$ simplifies in the case $U=\varnothing$ to

$$
d_{\varnothing}=\sum_{W \subseteq \mathbf{N}} c_{W} \bar{\Pi}_{W}(\beta)
$$

Now suppose that (i) and (ii) hold. To show that $\bar{P} \cdot \phi=\bar{Q} \cdot \lambda$, let $\alpha, \beta$ and $\alpha \lambda+\beta$ be defined as in (i) and the preceding derivation. Since by hypothesis $\phi$ and $\alpha \lambda+\beta$ denote the same sequence of scales, we may use (ii) and the identities in (5) above to conclude

$$
\begin{aligned}
\bar{P} \cdot \phi & =\sum_{W \subseteq \mathrm{N}} c_{W} \bar{\Pi}_{W}(\phi)=\sum_{W \subseteq \mathrm{N}} c_{W} \bar{\Pi}_{W}(\alpha \lambda+\beta) \\
& =\sum_{U \leq \mathrm{N}}\left[\bar{\Pi}_{U}(\alpha) \sum_{W \supseteq U} c_{W} \bar{\Pi}_{W-U}(\beta)\right] \bar{\Pi}_{U}(\lambda) \\
& =\sum_{U \subseteq \mathrm{N}} d_{U} \bar{\Pi}_{U}(\lambda)=\bar{Q} \cdot \lambda .
\end{aligned}
$$




\section{THREe FOP REPRESENTATION THEOREMS}

This section presents FOP generalizations of three well-known measurement representations, the utility representation of expected utility theory, the algebraic difference representation, and the additive conjoint representation. Although these generalizations differ in formal details, each makes use of the same strategy of axiomatization and proof. The informal proofs of the FOP representation theorems given here will emphasize the similarity of the role played by Lemmas 1 and 2 in establishing the existence of the representations. For the sake of simplicity, all representations will be restricted to the case where sign independence holds; extending the representations to the case where proper sign dependence can obtain is straightforward, but it requires certain formal complications that are not germane to the principal questions of interest. ${ }^{1}$ Fishburn and Keeney $(1974,1975)$ develop a FOP utility theory that takes into account the possibility of proper sign dependence. Their formal development applies, mutatis mutandis, to the other FOP theories described in this section.

\section{II(A). The FOP Utility Representation}

The basic constituents of expected utility (EU) theory are a set $K$ of consequences, a set $L$ of lotteries for consequences in $K$ and a preference order $\preccurlyeq_{p}$ among lotteries (Luce \& Raiffa, 1957). An empirical relational structure of EU theory is thus a triple $\left(L, K, \preccurlyeq_{p}\right)$. If the EU axioms as stated, for example, in Luce \& Raiffa (1957) hold of $\left(L, K, \preccurlyeq_{p}\right)$, then there exists an order preserving utility function $U: K \rightarrow \operatorname{Re}$ satisfying the expected utility hypothesis (cf. Luce \& Raiffa, 1957). Without attempting to state the EU representation theorem precisely, we pass to the essential point relating to the present work. The EU axioms imply that the utility representation $U$ is unique up to an affine transformation: if $U^{\prime}: K \rightarrow \operatorname{Re}$ is any other function satisfying the $\mathrm{EU}$ representation, then $U^{\prime}=\alpha U+\beta$ for some positive real $\alpha$ and real $\beta$.

Next, the utility representation will be generalized to a FOP utility representation (what Keeney \& Raiffa (1976) call a multilinear utility function). Suppose that the set of consequences $K$ is a Cartesian product $K=K_{1} \times K_{2} \times \cdots \times K_{N}$ of $N$ attributes and that the relational structure $\left(L, K, \preccurlyeq_{p}\right)$ satisfies the EU axioms. Hence, by the EU representation theorem, there exists an affinely unique utility representation $U$ for $\left(L, K, \preccurlyeq_{p}\right)$.

To formulate a FOP generalization of EU theory, one must first identify substructures of $\left(L, K, \preccurlyeq_{p}\right)$ that are built from lotteries in which only one attribute varies. For any $x \in K, i \in \mathbf{N}$ and $a \in K_{i}$, let $\left.x\right|_{i} a$ denote the sequence which is like $x$ expect that the $i$ th component has been replaced by $a$, let $K_{i x}$ denote the set $\left\{\left.x\right|_{i} b\right.$ : for some $\left.b \in K_{l}\right\}$, let $L_{i x} \subseteq L$ denote the subset of lotteries with consequences in $K_{i x}$, and let $\preccurlyeq_{i x}$ denote the restriction of $\preccurlyeq_{p}$ to $L_{i x}$. Evidently, the structure $\left(L_{i x}, K_{i x}, \preccurlyeq_{i x}\right)$ satisfies all the necessary properties of EU theory since it is a substructure of $\left(L, K, \preccurlyeq_{p}\right)$. Assuming that for any $i$ and $x$, the set $K_{i x}$ is sufficiently rich in the sense

${ }^{1}$ See Chap. 7 of Krantz et al. (1972) for definitions of independence and sign dependence. 
that the utility furction $U$ sends $K_{i x}$ onto an interval of real numbers, the structure $\left(L_{i x}, K_{i x}, \leqslant_{i x}\right)$ also satisfies nonnecessary, structural axioms of EU theory.

Since for lotteries in $L_{i x}$, only the outcome on the $i$ th factor varies, one may wish to regard $L_{i x}$ as a set of lotteries for outcomes in $K_{i}$ to be received or experienced along with the fixed consequences $\bar{x}_{l}$. This intuition may be formalized as follows. Let $L_{i}$ denote the set of lotteries with consequences in $K_{i}$. Define a function $\theta_{i x}: L_{i} \rightarrow L_{i x}$ by the condition that for any $g \in L_{i}, \theta_{i x}(g)$ is the lottery that results from $g$ when each outcome $a$ of $g$ is replaced by $\left.x\right|_{i} a$. Define an ordering $\preccurlyeq_{i x}^{*}$ of $L_{i}$ by

$$
g \preccurlyeq_{i x}^{*} g^{\prime} \quad \text { iff } \quad \theta_{i x}(g) \preccurlyeq_{i x} \theta_{i x}\left(g^{\prime}\right)
$$

for any $g, g^{\prime} \in L_{i}$. Evidently $\theta_{i x}$ is an isomorphism between the structure $\left(L_{i}, K_{i}, \preccurlyeq_{i x}^{*}\right)$ and $\left(L_{i x}, K_{i x}, \preccurlyeq_{i x}\right)$. Since $\left(L_{i x}, K_{i x}, \preccurlyeq_{i x}\right)$ satisfies EU theory, so must $\left(L_{i}, K_{i}, \leqslant_{i x}^{*}\right)$. Recalling that $U$ is a utility function for $\left(L, K, \leqslant_{p}\right)$, the restriction of $U$ to $K_{i x}$ (denoted $\left.U_{i x}\right)$ is a utility function for $\left(L_{i x}, K_{i x}, \leqslant_{i x}\right)$. Hence, the composition $U_{i x} \cdot \theta_{i x}$ is a utility function for $\left(L_{i}, K_{i}, \leqslant_{i x}^{*}\right)$.

Up to this point, we have only assumed that $\left(L, K, \preccurlyeq_{p}\right)$ satisfies EU theory and the technical assumption that $K_{i x}$ is sufficiently rich for any $i$ and $x$. The FOP utility representation is implied by a single additional postulate called uniattribute utility independence: for any $i \in \mathbf{N}$ and $x, y \in K$, the structures $\left(L_{i}, K_{i}, \preccurlyeq_{i x}^{*}\right)$ and $\left(L_{i}, K_{i}, \preccurlyeq_{i y}^{*}\right)$ are identical. This postulate is equivalent to asserting that the orderings $\preccurlyeq_{i x}^{*}$ and $\preccurlyeq_{i y}^{*}$ are the same.

Under these assumptions, an elementary proof of the FOP utility representation theorem can be given. Let $U$ be a utility function for $\left(L, K, \preccurlyeq_{p}\right)$ and choose any arbitrary fixed $y \in K$. Define a sequence $\phi=\left(\phi_{1}, \phi_{2}, \ldots, \phi_{N}\right)$ of scales by $\phi_{i}=U_{i y} \cdot \theta_{i y}$ for each $i \in \mathbf{N}$. As previously noted, $\phi_{i}=U_{i y} \cdot \theta_{i y}$ is an affinely unique representation for $\left(L_{i}, K_{i}, \preccurlyeq_{i y}^{*}\right)$. Moreover, since $K_{i y}$ is sufficiently rich, there exist $b, c \in K_{i}$ such that $U\left(\left.y\right|_{i} b\right) \neq U\left(\left.y\right|_{i} c\right)$, and thus, $\phi_{i}(b) \neq \phi_{i}(c)$ by definition of $\phi_{i}, U_{i y}$ and $\theta_{i y}$.

Consider any $x \in K . U_{i x} \cdot \theta_{i x}$ is a utility representation for $\left(L_{i}, K_{i}, \leqslant_{i x}^{*}\right)$ and uniattribute utility independence asserts that $\left(L_{i}, K_{i}, \leqslant_{i x}^{*}\right)$ and $\left(L_{i}, K_{i}, \leqslant_{i y}^{*}\right)$ are identical. Since $\phi_{i}$ is a utility representations for this latter structure, there exist real numbers $A_{i x}$ and $B_{i x}$ depending in general on $i$ and $x$ such that

$$
U_{i x} \cdot \theta_{i x}=A_{i x} \phi_{i}+B_{i x} .
$$

To see that the $A_{i x}$ and $B_{i x}$ satisfying (6) must be unique, suppose $\alpha_{i x}$ and $\beta_{i x}$ also satisfy (6) and choose $b, c \in K_{i}$ such that $\phi_{i}(b) \neq \phi_{i}(c)$. Then, $U_{i x} \cdot \theta_{i x}(b)-$ $U_{i x} \cdot \theta_{i x}(c)=A_{i x}\left[\phi_{l}(b)-\phi_{l}(c)\right]=\alpha_{i x}\left[\phi_{i}(b)-\phi_{i}(c)\right]$. Since $\phi_{i}(b)-\phi_{l}(c) \neq 0$, we have $A_{i x}=\alpha_{i x}$. Hence $B_{i x}=\beta_{i x}$. Moreover, note that if $\bar{x}_{i}=\bar{z}_{i}$ for any other $z \in K$, then $\left.x\right|_{i} a=\left.z\right|_{i} a$ for any $a \in K_{i}$. Hence $U_{i x}=U_{i z}$ and $\theta_{i x}=\theta_{i z}$; consequently, $U_{i x} \cdot \theta_{i x}=$ $U_{i z} \cdot \theta_{i z}$. Thus, the $A_{i x}$ and $B_{i x}$ satisfying (6) depend only on $i$ and $\bar{x}_{i}$.

Since the $A_{i x}$ and $B_{i x}$ satisfying (6) are unique and depend only on $i$ and $\bar{x}_{i}$, there 
exist well-defined functions $G_{i}: \bar{K}_{i} \rightarrow \operatorname{Re}$ and $H_{i}: \bar{K}_{i} \rightarrow \operatorname{Re}$ determined by the rule $G_{i}\left(\bar{x}_{i}\right)=A_{i x}$ and $H_{i}\left(\bar{x}_{i}\right)=B_{i x}$. But now we have the identity

$$
\begin{aligned}
U(x) & =U_{i x} \cdot \theta_{i x}\left(x_{i}\right)=A_{i x} \phi_{i}\left(x_{i}\right)+B_{i x} \\
& =G_{i}\left(\bar{x}_{i}\right) \phi_{i}\left(x_{i}\right)+H_{i}\left(\bar{x}_{i}\right)
\end{aligned}
$$

for any $x \in K$ and $i \in \mathbf{N}$. Thus by Definition 2, $U$ is multiaffine with respect to the sequence $\phi$ of scales. By Lemma 2, there exists a FOP $P$ such that $U=\bar{P} \cdot \phi$. Furthermore, the uniqueness of $P$ is as follows. If $U^{\prime}$ is any other utility function and $U^{\prime}=\bar{Q} \cdot \lambda$ for some FOP $Q$ and sequence $\lambda$ of scales, then there exist positive real $\alpha$ and real $\beta$ such that $U^{\prime}=\alpha U+\beta$. Hence, $\bar{Q} \cdot \lambda=\alpha(\bar{P} \cdot \phi)+\beta$, so the coefficients of $Q \cdot \lambda$ are related to the coefficients of $\alpha(P \cdot \phi)+\beta$ by Lemma 3 .

We have proved the following theorem. Let $K=K_{1} \times K_{2} \times \cdots \times K_{N}$ be a Cartesian product of arbitrary sets, and let $\left(L, K, \preccurlyeq_{p}\right)$ be an expected utility structure with utility function $U: K \rightarrow \operatorname{Re}$. We make the technical assumption that $U$ maps $K_{i x}$ onto an interval of real numbers for any $i \in \mathbf{N}$ and $x \in K$. In addition, we assume that $\left(L, K, \preccurlyeq_{p}\right)$ satisfies uniattribute utility independence: for any $i \in \mathbf{N}$, the preference ordering of lotteries for outcomes in $K_{i}$ to be received along with the fixed sequence $\bar{x}_{i}$ of outcomes on the remaining attributes does not depend on the choice of $\bar{x}_{i}$. Then, there exists a FOP $P$ and a sequence $\phi$ of scales such that $U=\bar{P} \cdot \phi$.

\section{II(B). The FOP Difference Representation}

Dyer and Sarin (1979) showed that the strategy of axiomatization used in generalizing EU theory to a FOP utility representation could also be applied to difference representations. The present exposition alters slightly their approach, but retains their basic method for axiomatizing the FOP difference representation.

By an algebraic difference structure, we mean a pair $\left(K \times K, \preccurlyeq_{d}\right)$, where $K$ is an arbitrary set and $\preccurlyeq_{d}$ is an ordering of $K \times K$, such that $\left(K \times K, \preccurlyeq_{d}\right)$ satisfies the axioms stated in Definition 3, Chap. 4 of Krantz et al. (1971, p. 151). By Theorem 4.2 of that work, if $\left(K \times K, \preccurlyeq_{d}\right)$ is an algebraic difference structure, there exists an affinely unique function $f: K \rightarrow \operatorname{Re}$ such that for any $w, x, y, z \in K$,

$$
w x \leqslant_{d} y z \quad \text { iff } f(w)-f(x) \leqslant f(y)-f(z),
$$

where $w x$ and $y z$ denote the ordered pairs $(w, x)$ and $(y, z)$, respectively. A function $f$ satisfying (7) is called an algebraic difference representation for $\left(K \times K, \preccurlyeq_{d}\right)$. Now suppose that $K$ is itself a Cartesian product of arbitrary sets, that is, $K=K_{1} \times K_{2} \times \cdots \times K_{N}$. We wish to establish conditions under which $f=\bar{P} \cdot \phi$ for some FOP $P$ and sequence $\phi$ of scales; when there exists an $f$ satisfying (7) such that $f=\bar{P} \cdot \phi$, then $P \cdot \phi$ will be called a FOP difference representation.

For any $i \in \mathbf{N}$ and $x \in K$, let $K_{i x}=\left\{\left.x\right|_{i} a\right.$ : for some $\left.a \in K_{i}\right\}$ and let $\preccurlyeq_{i x}$ denote the restriction of $\leqslant_{d}$ to $K_{i x} \times K_{i x}$. The structure $\left(K_{i x} \times K_{i x}, \preccurlyeq_{i x}\right)$ must satisfy all the necessary properties of an algebraic difference structure, since it is a substructure of $\left(K \times K, \preccurlyeq_{d}\right)$. Making the technical assumption that any $K_{i x}$ is sufficiently rich in the 
sense that $f$ sends $K_{i x}$ onto an interval of real numbers, $\left(K_{i x} \times K_{i x}, \preccurlyeq_{i x}\right)$ will also satisfy the nonnecessary, solvability axiom. Thus, $\left(K_{i x} \times K_{i x}, \leqslant_{i x}\right)$ is an algebraic difference structure and the restriction of $f$ to $K_{i x}$ (denoted $f_{i x}$ ) is an algebraic difference representation for $\left(K_{i x} \times K_{i x}, \leqslant_{i x}\right)$. Let $\leqslant_{i x}^{*}$ be an ordering of $K_{i} \times K_{i}$ defined by

$$
a b \preccurlyeq_{i x}^{*} c d \quad \text { iff } \quad\left(\left.x\right|_{i} a\right)\left(\left.x\right|_{i} b\right) \preccurlyeq_{i x}\left(\left.x\right|_{i} c\right)\left(\left.x\right|_{i} d\right)
$$

for any $a, b, c, d \in K_{i}$. Define a function $\theta_{i x}: K_{i} \rightarrow K_{i x}$ by $\theta_{i x}(a)=\left.x\right|_{i} a$ for any $a \in K_{i}$. Evidently, $\theta_{i x}$ is an isomorphism between $\left(K_{i} \times K_{i}, \leqslant_{i x}^{*}\right)$ and $\left(K_{i x} \times K_{i x}, \preccurlyeq_{i x}\right)$; hence, $\left(K_{i} \times K_{i}, \preccurlyeq_{i x}^{*}\right)$ is an algebraic difference structure and $f_{i x} \cdot \theta_{i x}$ is an algebraic difference representation for $\left(K_{i} \times K_{i}, \preccurlyeq_{i x}^{*}\right)$.

Now we are in a formally analogous position to the point in the utility axiomatization where the uniattribute utility independence axiom was proposed. The analogous postulate to be adopted here will be called mutual context invariance, and is stated as follows: for any $i \in \mathbf{N}$ and $x, y \in K$, the structures $\left(K_{i} \times K_{i}, \preccurlyeq_{i x}^{*}\right)$ and $\left(K_{i} \times K_{i}, \leqslant_{i y}^{*}\right)$ are identical. An equivalent statement of mutual context invariance can be given in the primitive terminology: for any $i \in \mathbf{N}, x, y \in K$ and $a, b, c, d \in K_{i}$,

$$
\left(\left.x\right|_{i} a\right)\left(\left.x\right|_{i} b\right) \preccurlyeq_{d}\left(\left.x\right|_{i} c\right)\left(\left.x\right|_{i} d\right) \quad \text { iff } \quad\left(\left.y\right|_{i} a\right)\left(\left.y\right|_{i} b\right) \preccurlyeq_{d}\left(\left.y\right|_{i} c\right)\left(\left.y\right|_{i} d\right) .
$$

It is easy to check that this qualitative axiom is equivalent to asserting that the orderings $\preccurlyeq_{i x}^{*}$ and $\preccurlyeq_{i y}^{*}$ are identical, for any $i \in \mathbf{N}$ and $x, y \in K$.

Adding mutual context invariance to the assumptions that $\left(K \times K, \leqslant_{d}\right)$ is an algebraic difference structure and that every $K_{i x}$ is sufficiently rich, we may now prove the FOP difference representation theorem. Let $f$ be an algebraic difference representation for $\left(K \times K, \preccurlyeq_{d}\right)$ and choose any arbitrary, fixed $y \in K$. Define a sequence $\phi=\left(\phi_{1}, \phi_{2}, \ldots, \phi_{N}\right)$ of scales by $\phi_{i}=f_{i y} \cdot \theta_{i y}$ for each $i \in \mathbf{N}$, where $f_{i y}$ is the restriction of $f$ to $K_{i y}$ and $\theta_{i y}$ is the function taking any $a \in K_{i}$ to $\left.y\right|_{i} a$. As previously noted, $\phi_{i}=f_{i y} \cdot \theta_{i y}$ is an affinely unique, algebraic difference representation for $\left(K_{i} \times K_{i}, \aleph_{i y}^{*}\right)$. Furthermore, since $K_{i y}$ is sufficiently rich, there exist $b, c \in K_{i}$ such that $U\left(\left.y\right|_{i} b\right) \neq U\left(\left.y\right|_{i} c\right)$, and thus, $\phi_{i}(b) \neq \phi_{i}(c)$ by definition of $\phi_{i}, f_{i y}$ and $\theta_{i y}$.

Consider any $x \in K$. Since $f_{i x} \cdot \theta_{i x}$ is an algebraic difference representation for $\left(K_{i} \times K_{i}, \preccurlyeq_{i x}^{*}\right)$ and mutual context invariance asserts that $\left(K_{i} \times K_{i}, \preccurlyeq_{i x}^{*}\right)$ and $\left(K_{i} \times K_{i}, \preccurlyeq_{i y}^{*}\right)$ are identical, we must have

$$
f_{i x} \cdot \theta_{i x}=A_{i x} \phi_{i}+B_{i x}
$$

for some positive real $A_{i x}$ and real $B_{i x}$. To see that $A_{i x}$ and $B_{i x}$ must be unique, suppose that $\alpha_{i x}$ and $\beta_{i x}$ also satisfy (8) and choose $b, c \in K_{i}$ such that $\phi_{i}(b) \neq \phi_{i}(c)$. Then, $f_{i x} \cdot \theta_{i x}(b)-f_{i x} \cdot \theta_{i x}(c)=A_{i x}\left[\phi_{i}(b)-\phi_{i}(c)\right]=\alpha_{i x}\left[\phi_{i}(b)-\phi_{i}(c)\right]$. Since $\phi_{i}(b)-$ $\phi_{i}(c) \neq 0$, we have $A_{i x}=\alpha_{i x}$. Hence $B_{i x}=\beta_{i x}$. Moreover, note that if $\bar{x}_{i}=\bar{z}_{i}$ for any other $z \in K$, then $\left.x\right|_{i} a=\left.z\right|_{i} a$ for any $a \in K_{i}$. Hence, $f_{i x}=f_{i z}$ and $\theta_{i x}=0_{i z}$; consequently, $f_{i x} \cdot \theta_{i x}=f_{i z} \cdot \theta_{i z}$, so the $A_{i x}$ and $B_{i x}$ satisfying (8) depend only on $i$ and $\bar{x}_{i}$. Thus, there are well-defined function $G_{i}: \bar{K}_{i} \rightarrow \operatorname{Re}$ and $H_{i}: \bar{K}_{i} \rightarrow \operatorname{Re}$ given by 
$G_{i}\left(\bar{x}_{i}\right)=A_{i x}$ and $H_{i}\left(\bar{x}_{i}\right)=B_{i x}$ for the $A_{i x}$ and $B_{i x}$ satisfying (8). But now, for any $x \in K$ and $i \in \mathbf{N}$, we have

$$
\begin{aligned}
f(x) & =f_{i x} \cdot \theta_{i x}\left(x_{i}\right)=A_{i x} \phi_{i}\left(x_{i}\right)+B_{i x} \\
& =G_{i}\left(\bar{x}_{i}\right) \phi_{i}\left(x_{i}\right)+H_{i}\left(\bar{x}_{i}\right) .
\end{aligned}
$$

By Definition 2, $f$ is multiaffine with respect to the sequence $\phi$ of scales. By Lemma 2, there exists a FOP $P$ such that $f=\bar{P} \cdot \phi$, and the FOP difference representation is established. The uniqueness of this representation is essentially the same as that of the FOP utility representation stated near the end of subsection I(A).

\section{II(C). The Additive Representation with FOP Components}

The FOP generalizations of the utility and algebraic difference representations depend crucially on the interval scale uniqueness of the representations. Since additive conjoint measurement also leads to interval scales on its components, one might expect that it too possesses a FOP generalization. This is indeed the case. The material in this subsection II(C) can be omitted on first reading for it will not be built upon in later discussions.

Here, we will solve the following problem. Let $\Gamma=\Gamma_{1} \times \Gamma_{2} \times \cdots \times \Gamma_{N}$ and $\Delta=\Delta_{1} \times \Delta_{2} \times \cdots \times \Delta_{M}$ be Cartesian products of arbitrary sets (for $N, M \geqslant 1$ ), and let $\preccurlyeq_{e}$ be an ordering of $\Gamma \times \Delta$. Find conditions under which there exist sequences of scales $\phi=\left(\phi_{1}, \phi_{2}, \ldots, \phi_{N}\right)$ and $\eta=\left(\eta_{1}, \eta_{2}, \ldots, \eta_{M}\right)$ and FOPs $P$ and $Q$ such that for every $u x, v y \in \Gamma \times \Delta$

$$
u x \preccurlyeq_{e} v y \quad \text { iff } \quad \bar{P} \cdot \phi(u)+\bar{Q} \cdot \eta(x) \leqslant \bar{P} \cdot \phi(v)+\bar{Q} \cdot \eta(y) .
$$

If $\left(\Gamma \times \Delta, \preccurlyeq_{e}\right)$ has a representation of the form (9), it will be said to have an additive representation with FOP components.

In order to achieve the desired generalization of additive conjoint measurement, we will need to make use of properties of symmetric additive conjoint structures, where an additive conjoint structure $\left(\Gamma \times \Delta, \preccurlyeq_{e}\right)$ is symmetric iff for any $u, v \in \Gamma$ there exists $y, z \in \Delta$ such that $u y \sim_{e} v z$ and for any $w, x \in \Delta$ there exist $s, t \in \Gamma$ such that $s w \sim_{e} t x$. We will need to use of the following relationship between symmetric additive conjoint structures and algebraic difference structures.

Lemma 4. Let $\left(\Gamma \times \Delta, \preccurlyeq_{e}\right)$ be any symmetric additive conjoint structure (see Krantz et al., 1971, Definition 7, Chap. 6, p. 256). Then $(I \times \Gamma, R)$ and $(\Delta \times \Delta, T)$ are algebraic difference structures, where the orderings $R$ and $T$ are defined by

(i) For any $s, t, u, v \in \Gamma$, st $R$ uv iff $s y \leqslant_{e} t z$ and $v z \leqslant_{e} u y$ for some $y, z \in \Delta$.

(ii) For any $w, x, y, z \in \Delta, w x T y z$ iff $v w \preccurlyeq_{e} u x$ and $u z \preccurlyeq_{e} v y$ for some $u, v \in \Gamma$.

Since $\left(\Gamma \times A, \preccurlyeq_{e}\right)$ is an additive conjoint structure, it has an additive representation and therefore satisfies triple cancellation (as defined in Chap 6 of Krantz et al., 
1971). The proof that $(\Gamma \times \Gamma, R)$ and $(\Delta \times \Delta, T)$ satisfy the algebraic difference axioms follows from triple cancellation and the symmetric additive conjoint axioms. Chapter 6 of Krantz et al. (1971) discusses the relationship between the symmetric additive conjoint structure and the positive difference structure, and their discussion can be applied with only slight changes to the algebraic difference structure.

The following corollary to Lemma 4 will be useful.

COROLlary 4.1. Let $\left(\Gamma \times \Delta, \preccurlyeq_{e}\right)$ be a symmetric additive conjoint structure and let $F: \Gamma \rightarrow \operatorname{Re}$ and $S: \Delta \rightarrow \operatorname{Re}$ be the scales of an additive representation of $\left(\Gamma \times \Delta, \leqslant_{e}\right)$. Then $F$ is an algebraic difference representation for $(\Gamma \times \Gamma, R)$ and $S$ is an algebraic difference representation for $(\Delta \times \Delta, T)$.

The proof of the corollary is an elementary consequence of the definitions of the structures $(\Gamma \times \Gamma, R)$ and $(\Delta \times \Delta, T)$.

These relationships between additive conjoint and algebraic difference measurament will be crucial to the FOP generalization of additive conjoint measurement, to which we now turn. Let $\Gamma=\Gamma_{1} \times \Gamma_{2} \times \cdots \times \Gamma_{N}$ and $\Delta=\Delta_{1} \times \Delta_{2} \times \cdots \times \Delta_{M}$ be Cartesian products of arbitrary sets (for $N, M \geqslant 1$ ) and let $\preccurlyeq_{e}$ be an ordering of $\Gamma \times \Delta$. We assume that $\left(\Gamma \times \Delta, \preccurlyeq_{e}\right)$ is an additive conjoint structure, and hence, there exist functions $F: \Gamma \rightarrow \operatorname{Re}$ and $S: \Delta \rightarrow \operatorname{Re}$ such that

$$
u x \preccurlyeq_{e} v y \quad \text { iff } \quad F(u)+S(x) \leqslant F(v)+S(y)
$$

for any $u, v \in \Gamma$ and $x, y \in \Delta$.

For any $u \in \Gamma$ and $i \in \mathbf{N}$, let $\Gamma_{i u}=\left\{\left.u\right|_{i} a\right.$ : for some $\left.a \in \Gamma_{i}\right\}$ and for any $x \in \Delta$ and $j \in \mathbf{M}$, let $\Delta_{j x}=\left\{\left.x\right|_{j} p\right.$ : for some $\left.p \in{ }_{j}\right\}$. Let $\preccurlyeq_{i j u x}$ be the restriction of $\leqslant_{e}$ to $\Gamma_{l u} \times \Delta_{j x}$. Since $\left(\Gamma_{i u} \times \Delta_{j x}, \preccurlyeq_{i j u x}\right)$ is a substructure of $\left(\Gamma \times \Delta, \preccurlyeq_{e}\right)$, it must satisfy all the necessary axioms of the additive conjoint structure. We assume that any substructure $\left(\Gamma_{i u} \times \Delta_{j x}, \preccurlyeq_{i j u x}\right)$ is symmetric, and in addition, we make the technical assumption that $F$ maps $\Gamma_{i u}$ and $S$ maps $\Delta_{j x}$ onto intervals of real numbers $\left(\Gamma_{i u}\right.$ and $\Delta_{j x}$ are sufficiently rich). Under this last assumption, $\left(\Gamma_{i u} \times \Delta_{j x}, \preccurlyeq_{i j u x}\right)$ also satisfies the additive conjoint solvability condition and the assumption that each component is essential (i.e., nontrivial). Thus, $\left(\Gamma_{i u} \times \Delta_{j x}, \preccurlyeq_{i j u x}\right)$ is a symmetric additive conjoint structure. Let $F_{i u}$ denote the restriction of $F$ to $\Gamma_{i u}$ and let $S_{j x}$ denote the restriction of $S$ to $\Delta_{j x} . F_{i u}$ and $S_{j x}$ are the component scales of an additive representation for $\left(\Gamma_{i u} \times \Delta_{j x}, \leqslant_{i j u x}\right)$.

Since $\left(\Gamma_{i u} \times \Delta_{j x}, \preccurlyeq_{i j u x}\right)$ is a symmetric additive conjoint structure, one can define algebraic difference structures $\left(\Gamma_{i u} \times \Gamma_{i u}, R_{l j u x}\right)$ and $\left(\Delta_{j x} \times \Delta_{j x}, T_{i j u x}\right)$ as in Lemma 4. Define functions $\theta_{i u}: \Gamma_{i} \rightarrow \Gamma_{i u}$ and $\lambda_{j x}: \Delta_{j} \rightarrow \Delta_{j x}$ by

$$
\begin{array}{ll}
\theta_{i u}(a)=\left.u\right|_{i} a & \text { for any } a \in \Gamma_{i}, \\
\lambda_{j x}(p)=\left.x\right|_{j} p & \text { for any } p \in \Delta_{j} .
\end{array}
$$


Define orderings $R_{i j u x}^{*}$ and $T_{i j u x}^{*}$ of $\Gamma_{i} \times \Gamma_{i}$ and $\Delta_{j} \times \Delta_{j}$, respectively, by

$$
\begin{array}{cc}
a b R_{i j u x}^{*} c d & \text { iff } \theta_{i u}(a) \theta_{i u}(b) R_{i j u x} \theta_{i u}(c) \theta_{i u}(d), \\
p q T_{i j u x}^{*} r s & \text { iff } \quad \lambda_{j x}(p) \lambda_{j x}(q) T_{i j u x} \lambda_{j x}(r) \lambda_{j x}(s) .
\end{array}
$$

Evidently, $\left(\Gamma_{i} \times \Gamma_{i}, R_{i j u x}^{*}\right)$ and $\left(\Delta_{j} \times \Delta_{j}, T_{i j u x}^{*}\right)$ are algebraic difference structures that are isomorphic to $\left(\Gamma_{i u} \times \Gamma_{i u}, R_{i j u x}\right)$ and $\left(\Delta_{j x} \times \Delta_{j x}, T_{i j u x}\right)$ under the respective isomorphisms $\theta_{i u}$ and $\lambda_{j x}$.

We are now able to state the key axiom needed for the desired generalization of additive conjoint measurement. As with the previous generalizations of utility and algebraic difference representations, the required axiom is equivalent to asserting that unidimensional substructures are invariant with respect to changes in the stimulus context defining the substructure.

\section{Axiom of Mutual Context Invariance for Additive Conjoint Intervals:}

(i) For any $i \in \mathbf{N}$, if for some $u \in \Gamma, x, y \in \Delta$ and $a, b, c, d \in \Gamma_{i}$ the relations $\left(\left.u\right|_{i} a\right) x \preccurlyeq_{e}\left(\left.u\right|_{i} b\right) y$ and $\left(\left.u\right|_{i} d\right) y \preccurlyeq_{e}\left(\left.u\right|_{i} c\right) x$ hold, then for any $v \in \Gamma$ and $x^{\prime}, y^{\prime} \in \Delta$, if $\left(\left.v\right|_{i} a\right) x^{\prime} \geqslant_{e}\left(\left.v\right|_{i} b\right) y^{\prime}$, then $\left(\left.v\right|_{i} c\right) x^{\prime} \geqslant_{e}\left(\left.v\right|_{i} d\right) y^{\prime}$.

(ii) For any $j \in \mathbf{M}$, if for some $x \in \Delta, u, v \in \Gamma$ and $p, q, r, s \in \Delta_{j}$ the relations $u\left(\left.x\right|_{j} p\right) \leqslant_{e} v\left(\left.x\right|_{j} q\right)$ and $v\left(\left.x\right|_{j} s\right) \leqslant_{e} u\left(\left.x\right|_{j} r\right)$, then for any $y \in \Delta$ and $u^{\prime}, v^{\prime} \in \Gamma$, if $u^{\prime}\left(\left.y\right|_{j} p\right) \geqslant_{e} v^{\prime}\left(\left.y\right|_{j} q\right)$, then $u^{\prime}\left(\left.y\right|_{j} r\right) \geqslant_{e} v^{\prime}\left(\left.y\right|_{j} s\right)$.

Part (i) of the axiom implies that $\left(\Gamma_{i} \times \Gamma_{i}, R_{i j u x}^{*}\right)$ and $\left(\Gamma_{i} \times \Gamma_{i}, R_{i k v y}^{*}\right)$ are identical for any $i \in \mathbf{N}, j, k \in \mathbf{M}, u, v \in \Gamma$ and $x, y \in \Delta$. To see this, suppose that $a b R_{i j u x}^{*} c d$ for some $a, b, c, d \in \Gamma_{i}$. By definition of $R_{i j u x}^{*},\left(\left.u\right|_{i} a\right)\left(\left.u\right|_{i} b\right) R_{i j u x}\left(\left.u\right|_{i} c\right)\left(\left.u\right|_{i} d\right)$. Using the definition of $R_{i j u x}$ by means of Lemma 4 , we know there exist $p, q \in \Delta_{j}$ such that $\left(\left.u\right|_{i} a\right)\left(\left.x\right|_{j} p\right) \preccurlyeq_{e}\left(\left.u\right|_{i} b\right)\left(\left.x\right|_{j} q\right)$ and $\left(\left.u\right|_{i} d\right)\left(\left.x\right|_{j} q\right) \preccurlyeq_{e}\left(\left.u\right|_{i} c\right)\left(\left.x\right|_{j} p\right)$. For any other $k \in \mathbf{M}$, $y \in \Delta$ and $v \in \Gamma$, we know that there exist $r, s \in \Delta_{k}$ such that $\left(\left.v\right|_{i} a\right)\left(\left.y\right|_{k} r\right) \sim_{e}$ $\left(\left.v\right|_{i} b\right)\left(\left.y\right|_{k} s\right)$, because $\left(\Gamma_{i w} \times \Delta_{k y}, \preccurlyeq_{i k v y}\right)$ is symmetric. Therefore $\left(\left.v\right|_{i} c\right)\left(\left.y\right|_{k} r\right) \geqslant_{e}$ $\left(\left.v\right|_{i} d\right)\left(\left.y\right|_{k} s\right)$ by part (i) of the axiom. By the definition of $R_{i k t y}$, we have $\left(\left.v\right|_{i} a\right)\left(\left.v\right|_{i} b\right) R_{i k v y}\left(\left.v\right|_{i} c\right)\left(\left.v\right|_{i} d\right)$. Thus, $a b R_{i k v y}^{*} c d$ and consequently, $R^{*}{ }_{i j u x}$ and $R_{i k i y}^{*}$ are identical orderings. This proves that $\left(\Gamma_{i} \times \Gamma_{i}, R_{i j u x}^{*}\right)$ and $\left(\Gamma_{i} \times \Gamma_{i}, R_{i k t y}^{*}\right)$ are identical. The proof that $\left(\Delta_{j} \times \Delta_{j}, T_{i j u x}^{*}\right)$ and $\left(\Delta_{j} \times \Delta_{j}, T_{n j v y}^{*}\right)$ are identical for any $i, n \in \mathbf{N}, j \in \mathbf{M}, u, v \in \Gamma$, and $x, y \in \Delta$ is completely analogous to the preceding argument.

But now it is easy to show that the function $F: \Gamma \rightarrow \operatorname{Re}$ in the additive representation of $\left(\Gamma \times \Delta, \preccurlyeq_{e}\right)$ is represented by a FOP. Choose any $u \in \Gamma_{i}$ and let $\phi_{i}=F_{i u} \cdot \theta_{i u}$ for every $i \in \mathbf{N}$. Note that $\phi_{i}$ is an algebraic difference representation for $\left(\Gamma_{i} \times \Gamma_{i}, R_{i j u x}^{*}\right)$ since $F_{i u}$ is an algebraic difference representation for $\left(\Gamma_{i u} \times \Gamma_{i u}, R_{i j u x}\right)$ by Corollary 4.1 and $\theta_{i u}$ is an isomorphism $(j \in \mathbf{M}$ and $x \in \Delta$ may be chosen arbitrarily). But then for any $v \in \Gamma, k \in \mathbf{M}$, and $y \in \Delta, F_{i v} \cdot \theta_{i v}$ is an algebraic difference representation for $\left(\Gamma_{i} \times \Gamma_{i}, R_{i k v y}^{*}\right)$, and this structure and $\left(\Gamma_{i} \times \Gamma_{i}, R_{i j u x}^{*}\right)$ are identical. By the uniqueness of the algebraic difference representation

$$
F_{i v} \cdot \theta_{i v}=A_{i v} \phi_{i}+B_{i v}
$$


for $A_{i v}$ a positive real and $B_{i v}$ real. By arguments similar to those given in subsections $\mathrm{II}(\mathrm{A})$ and $\mathrm{II}(\mathrm{B})$, the $A_{i v}$ and $B_{i v}$ satisfying (10) are unique and depend only on $i$ and $\bar{v}_{i}$. Hence, we may define functions $G_{i}: \bar{\Gamma}_{i} \rightarrow \operatorname{Re}$ and $H_{i}: \bar{\Gamma}_{i} \rightarrow \operatorname{Re}$ by $G_{i}\left(\bar{v}_{i}\right)=A_{i r}$ and $H_{i}\left(\bar{v}_{i}\right)=B_{i v}$. But then, for any $v \in \Gamma$,

$$
\begin{aligned}
F(v) & =F_{i v} \cdot \theta_{i v}\left(v_{i}\right)=A_{i v} \phi_{i}\left(v_{i}\right)+B_{i v} \\
& =G_{i}\left(\bar{v}_{i}\right) \phi_{i}\left(v_{i}\right)+H_{i}\left(\bar{v}_{i}\right) .
\end{aligned}
$$

Thus, $F$ is multiaffine with respect to the sequence $\phi$ of scales. By Lemma 2 , there exists a FOP $P$ such that $F=\bar{P} \cdot \phi$. A similar argument using part (ii) of the axiom shows that $S=\bar{Q} \cdot \eta$ for some FOP $Q$ and sequence $\eta$ of scales.

To summarize, we have proved the following. Suppose $\Gamma=\Gamma_{1} \times \Gamma_{2} \times \cdots \times \Gamma_{N}$, $\Delta=\Delta_{1} \times \Delta_{2} \times \cdots \times \Delta_{M}$ and $\xi_{e}$ is an ordering of $\Gamma \times \Delta$ (for $\left.N, M \geqslant 1\right)$. If $\left(I \times \Delta_{,} \preccurlyeq_{e}\right)$ is an additive conjoint structure, every $\left(\Gamma_{i u} \times \Delta_{j x}, \preccurlyeq_{i j u x}\right)$ is symmetric, every $\Gamma_{i u}$ and $\Delta_{j x}$ is sufficiently rich, and the two parts to the axiom of mutual context invariance for additive conjoint intervals hold, then there exist FOPs $P$ and $Q$ and sequences $\phi$ and $\eta$ of scales such that the representation (9) holds for all $u, v \in \Gamma$ and $x, y \in \Delta$. The assumptions that every $\left(\Gamma_{i u} \times \Delta_{j x}, \preccurlyeq_{i j u x}\right)$ is symmetric and that $F_{i u}$ maps $\Gamma_{i u}$ and $S_{j x}$ maps $\Delta_{j x}$ onto intervals of real numbers should be weakened. The required formal analysis lies beyond the scope of the present essay.

\section{II(D). Relation to Previous Work on FOP Representations}

The proofs presented here reduce the existence of FOP representations to Lemmas 1 and 2. The present essay appears to be the first explicit statement and proof of these lemmas, although Keeney and Raiffa are clearly aware of the principle that they formalize (cf. Keeney, 1972; Keeney and Raiffa, 1976). The analysis of the uniqueness of these representations given in Lemmas 1-3 is new, so far as we know.

The utility axiom which is called uniattribute utility independence in this essay was formulated by Keeney (1968) in a study of the two attribute case. The axiom was called strong conditional utility independence in Raiffa's discussion of the two attribute case (Raiffa, 1969). Keeney (1972) generalized the axiom and representation to the $N$ attribute case, calling the axiom mutual utility independence. Later, Keeney and Raiffa (1976) used "mutual utility independence" to refer to a different, stronger property. Although they use the axiom of uniattribute utility independence to derive the general $N$ attribute FOP utility representation (multilinear utility function), they do not adopt any specific name for this axiom. The various derivations of the FOP utility representation produced by Keeney and Raiffa proceed by describing a scaling algorithm that yields a specific FOP utility representation. They do not discuss the uniqueness of this representation nor the general principle that a multiaffine function has a FOP representation.

Dyer and Sarin (1979) noticed that a difference representation could be generalized to a FOP difference representation if every attribute of a Cartesian product was weakly difference independent of all other attributes. This condition is essentially the axiom of mutual context invariance defined in subsection II(B). They point out that 
this condition implies that a difference representation must be multiaffine, or conditionally cardinal, in their terminology. Noting that conditional cardinality is the key property for the derivation of the FOP utility representation in Keeney and Raiffa (1976), they assert the existence of a FOP difference representation without proof and also without establishing its uniqueness. Dyer and Sarin apply the theory of FOP difference representations to the measurement of riskless value, as developed in Ellsberg (1954).

The generalization of additive conjoint measurement described in subsection II(C) appears to be new, although it is a straightforward continuation of the formal approach developed by Keeney, Raiffa, Dyer, and Sarin.

\section{Context Invariance and the Multiattribute GENERALIZATION OF RELATION THEORY}

The previous section has presented three FOP generalizations of well-known measurement theories. Each of these generalizations depends on a postulate asserting, in essence, that uniattribute substructures are invariant with respect to changes in the fixed levels of the remaining attributes. Next, it will be argued that this kind of invariance postulate has a profound psychological interpretation.

To develop this point, we will study the multiattribute generalization of a theory of subjective magnitude proposed by Roger Shepard (1981) and David Krantz (1972) to account for empirically established consistencies among so-called "direct" judgments of subjective magnitude. This theory, called Relation theory, is primarily concerned with the psychological structure of uniattribute magnitudes (magnitudes depending on the variation of a single physical parameter) or relations among uniattribute magnitudes. But since Relation theory is built upon the algebraic difference structure, the theory can easily be generalized to multiattribute magnitudes (magnitudes depending on the variation of several physical parameters) using the FOP difference theory developed in the previous section. The following exposition of Relation theory borrows extensively from Shepard (1981) and Krantz (1972) but deviates from their formulation in that little emphasis will be placed on the operation of cross-modality matching. Moreover, no attempt will be made to compare Relation theory to alternative theories of subjective magnitude which are discussed in Shepard and Krantz.

According to Relation theory, subjective magnitude is a psychological property of pairs of stimuli. The fundamental or essential structure of subjective magnitude is the ordering of stimulus pairs by the magnitude of their sensation relations on a specified subjective dimension. The hypothesis that sensation constitutes a psychological quantity is justified in Relation theory by postulating that the ordering of stimulus pairs satisfies axiomatic properties implying the existence of a numerical representation of the pair ordering by the ordering of ratios of scale values.

More formally, let $K$ be any sensory continuum, that is, a set of stimuli that can be ordered with respect to some attribute such as loudness, brightness, or perceived 
length. Although this is not a necessary assumption in Relation theory, it will be assumed here that the sensory continuum is physically parametrized and that subjective magnitude is a continuous, increasing function of physical intensity. The magnitude ordering $\preccurlyeq_{m}$ of stimulus pairs in $K \times K$ can be determined by any of a variety of psychophysical methods including the direct comparison of stimulus pairs, so-called "ratio estimation" or "ratio production" procedures, or cross-modality matching. Relation theory postulates that the empirical structure $\left(K \times K, \preccurlyeq_{m}\right)$ determined by some method of magnitude judgment satisfies the algebraic difference axioms. Thus, Relation theory proposes that sensation magnitude can be represented by a scale $\psi: K \rightarrow \operatorname{Re}^{+}$satisfying

$$
a b \preccurlyeq_{m} c d \quad \text { iff } \quad \psi(a) / \psi(b) \leqslant \psi(c) / \psi(d)
$$

for all $a, b, c, d \in K\left(\mathrm{Re}^{+}=\right.$the positive real numbers $)$.

Several remarks must be made concerning this formulation of Relation theory. First, the use of numerical ratios in the representation of $\left(K \times K, \preccurlyeq_{m}\right)$ is purely conventional, depending only on convenience and familiarity. The scale $\log (\psi)$ provides a difference representation of $\left(K \times K, \preccurlyeq_{m}\right)$ that is empirically and theoretically equivalent. Indeed, the class of equivalent alternative numerical representations is quite broad (Krantz et al., 1971, p. 152). Second, since the $\log (\psi)$ difference representation is affinely unique, $\psi$ is a $\log$ interval scale (Stevens, 1957; Krantz, 1972)-if $\psi^{\prime}$ also satisfies (11), then $\psi^{\prime}=\beta \psi^{\alpha}$ for positive real $\alpha$ and $\beta$. It should be mentioned that if the requirements imposed on the numerical representation are strengthened as in Krantz's theory of cross-modality matching, then ratio scale uniqueness of the representation can be established (Krantz, 1972). But within the weaker version of Relation theory developed here, only log interval uniqueness is achieved. Third, a principal motive for developing Relation theory is that several well-known alternative methods of magnitude judgment yield mutually consistent results (Krantz, 1972), suggesting that the same $\preccurlyeq_{m}$ ordering could be determined by different psychophysical methods including, but not necessarily restricted to, the various methods of "direct" magnitude described by Stevens $(1957,1975)$ and Marks (1974). Indeed, part of the justification for claiming to have determined the structure of sensation magnitude lies in the mutually consistent results obtained by operationally distinct psychophysical procedures. Although the present formulation does not specify the psychophysical procedure used in determining the $\preccurlyeq_{m}$ ordering, it may be assumed to be any method that yields results consistent with ratio estimation (cf. Krantz, 1972).

The generalization of Relation theory to FOP ratio representation of multiattribute magnitude is formally a straightforward application of the results of subsection II(B), but here we will emphasize the psychophysical interpretation of the mathematical structures appearing in that theory. For the sake of concreteness, suppose we are concerned with loudness judgments for complex combinations of pure tones. Let $K_{i}$ denote a set of pure tones varying in intensity at some fixed frequency $\eta_{i}$. Letting $K=K_{1} \times K_{2} \times \cdots \times K_{N}$, any $x \in K$ is an acoustical stimulus with 
intensity $x_{i}$ at frequency $\eta_{i}$. A psychophysical procedure like ratio estimation can be used to determine an ordering $\preccurlyeq_{m}$ of $K \times K$. If Relation theory is valid theory of multiattribute magnitude, the structure $\left(K \times K, \preccurlyeq_{m}\right)$ should be an algebraic difference structure-loudness relations among complex stimuli are ordered like ratios of numerical scale values.

Now consider uniattribute magnitude substructures of the form $\left(K_{i x} \times K_{i x}, \leqslant_{i x}\right)$ as defined in subsection II(B). The ordering $\preccurlyeq_{i x}$ is simply the loudness magnitude ordering $\preccurlyeq_{m}$ applied to stimulus pairs that vary in intensity only at the frequency $\eta_{i}$, the remaining frequencies being held fixed at $\bar{x}_{i}$. Thus, $\bar{x}_{i}$ may be interpreted as the fixed stimulus context for a loudness experiment in which only the $i$ th frequency varies in intensity. Since each context $\bar{x}_{i}$ determines a different uniattribute substructure, there are many such substructures. Furthermore, no a priori constraint relates the fundamental magnitude orderings, $\preccurlyeq_{i x}$ and $\preccurlyeq_{i y}$, for any two distinct substructures $\left(K_{i x} \times K_{i x}, \preccurlyeq_{i x}\right)$ and $\left(K_{i y} \times K_{i y}, \preccurlyeq_{i y}\right)$.

The mutual context invariance axiom, the key to the FOP generalization of Relation theory, may be interpreted in either of two ways. First, since only the intensity at $\eta_{i}$ varies in the magnitude substructure $\left(K_{i x} \times K_{i x}, \preccurlyeq_{i x}\right)$, it may seem more natural to regard the stimuli as elements of the form $a \in K_{i}$ rather than of the form $\left.x\right|_{i} a \in K_{i x}$. To formalize this intuition, one proceeds as in the previous section. Define the function $\theta_{i x}: K_{i} \rightarrow K_{i x}$ by $\theta_{i x}(a)=\left.x\right|_{i} a$, and let $\left(K_{i} \times K_{i}, \leqslant_{i x}^{*}\right)$ denote the structure isomorphic to $\left(K_{i x} \times K_{i x}, \preccurlyeq_{i x}\right)$ under $\theta_{i x}$. Since $\left(K_{i} \times K_{i}, \preccurlyeq_{i x}^{*}\right)$ and $\left(K_{i x} \times K_{i x}, \preccurlyeq_{i x}\right)$ are really just alternative formalisms for describing the same loudness experiment, we may interpret the ordering $\preccurlyeq_{i x}^{*}$ as the fundamental magnitude ordering for stimuli at frequency $\eta_{i}$ when presented in the context $\bar{x}_{i}$. Mutual context invariance asserts that orderings $\leqslant_{i x}^{*}$ and $\leqslant_{i y}^{*}$ are identical for any $x, y \in K$ and $i \in \mathbf{N}$. Hence, in keeping with its name, mutual context invariance asserts that for every $i$, the fundamental magnitude ordering $\preccurlyeq_{i x}^{*}$ for stimuli varying only at frequency $\eta_{i}$ is invariant with respect to changes in the stimulus context $\bar{x}_{i}$.

A deeper mathematical understanding of mutual context invariance can be derived from a formal development that has been omitted from the present discussion, but its outlines will be briefly sketched. We say that the pair $x y \in K \times K$ is a positive interval if $x x<_{m} x y$. For any positive interval $x y$, the equivalence class of $x y$ is defined to be the set of pairs $z w$ satisfying $x y \sim_{m} z w$. By combining Definition 4.4 (p. 151) with Definition 4.2 (p. 147) of Krantz et al. (1971), it can be shown that there exists a natural binary operation under which the set of equivalence classes of an algebraic difference structure can be made into a semigroup. For any $x, y \in K$, the uniattribute substructures $\left(K_{i x} \times K_{i x}, \preccurlyeq_{i x}\right)$ and $\left(K_{i y} \times K_{i y}, \preccurlyeq_{i y}\right)$ are both algebraic difference structures and hence, their associated equivalence classes are both semigroups. The essence of the mutual context invariance postulate is as follows. Let $h$ be the map taking the equivalence class of $\left(\left.x\right|_{i} a\right)\left(\left.x\right|_{i} b\right)$ to the equivalence class of $\left(\left.y\right|_{i} a\right)\left(\left.y\right|_{i} b\right)$. The postulate of mutual context invariance guarantees that $h$ is a welldefined semigroup isomorphism.

If mutual context invariance holds, we know from the previous section that there exists a FOP $P$ and a sequence $\phi$ of scales such that $P \cdot \phi$ is an algebraic difference 
representation for $\left(K \times K, \preccurlyeq_{m}\right)$. Hence, $e^{P \cdot \phi}$ is a ratio representation for $\left(K \times K, \preccurlyeq_{m}\right)$ in the sense that for any $w, x, y, z \in K$,

$$
w x \leqslant_{m} y z \quad \text { iff } \quad e^{\bar{P} \cdot \phi(x)} / e^{\bar{P} \cdot \phi(x)} \leqslant e^{\bar{P} \cdot \phi(y)} / e^{\bar{P} \cdot \phi(z)} .
$$

The representation (12) will be called a FOP ratio representation.

The FOP ratio representation is no mere technical device or mathematical amusement. Rather, it embodies a profound psychophysical theory of the perception of magnitude for multiattributed stimuli. Relation theory postulates that the fundamental structure of subjective magnitude is the ordering of stimulus pairs according to the magnitude of their sensation relations on a specified subjective dimension. When the stimuli are multiattributed, the multiattribute magnitude contains many uniattribute magnitudes as substructures. The FOP ratio representation is equivalent to asserting that the fundamental structure of every uniattribute magnitude is invariant with respect to changes in the stimulus context. The FOP ratio representation can thus be seen to characterize the psychophysical law for any multiattribute magnitude whose constituent uniattribute magnitudes are mutually context invariant (in both the formal and intuitive meaning of context invariance).

\section{Power Ratio Laws}

Krantz (1972) and Shepard (1981) point out that the power law for subjective magnitude has a simple axiomatization if Relation theory is assumed. Here, we will state their axiomatization and then show that it provides the basis for an interesting multiattribute generalization of the power law using FOP representation theory.

Let $K$ be a sensory continuum and let $\preccurlyeq_{m}$ be an empirical ordering of $K \times K$. As before, it will be assumed that the sensory continuum is physically parametrized and that subjective magnitude is a continuous, increasing function of physical intensity. Since each stimulus $x \in K$ is identified by its measure in physical units, numerical operations on $x$ like scalar multiplication, addition of a constant or raising to a power are well defined. We say that $\left(K \times K, \preccurlyeq_{m}\right)$ has a power ratio representation provided that there exists a real positive $\beta$ such that

$$
w x \preccurlyeq_{m} y z \quad \text { iff } \quad w^{\beta} / x^{\beta} \leqslant y^{\beta} / z^{\beta}
$$

for all $w, x, y, z \in K$.

According to Relation theory, $\left(K \times K, \preccurlyeq_{m}\right)$ satisfies the algebraic difference axioms. The existence of a power ratio representation is equivalent to adding the following axiom to the algebraic difference axioms.

Uniattribute Power Ratio Axiom. For every $x, y \in K$ and positive real $t$,

$$
x y \sim_{m}(t x)(t y) .
$$

It is easy to show that the power ratio representation (13) implies (14). To prove the 
converse implication, let $\psi: K \rightarrow \mathrm{Re}^{+}$be a scale that represents $\preccurlyeq_{m}$ as numerical ratios. By (14), $\psi$ satisfies

$$
\psi(x) / \psi(y)=\psi(t x) / \psi(t y) .
$$

Setting $y=1$ and multiplying both sides of the identity by $\psi(t) / \psi(1)$ yields

$$
\psi(t x) / \psi(1)=(\psi(t) / \psi(1))(\psi(x) / \psi(1)) .
$$

Defining the function $h$ by $h(x)=\psi(x) / \psi(1)$, we have $h(t x)=h(t) h(x)$, this last identity being one of the basic Cauchy functional equations (Aczel, 1966). The only continuous, increasing solution to this equation is $h(x)=x^{\beta}$ for some positive real $\beta$. Thus, $\psi(x)=\alpha x^{\beta}$, where $\alpha=\psi(1)$. Since $\psi$ is a ratio representation for $\preccurlyeq_{m}$, the power ratio representation (13) holds.

The present exposition of the power ratio representation is much less interesting than that of Krantz (1972) or Shepard (1981) because it leaves out the structure of cross-modality matching. Without taking cross-modality matching into consideration, the identity function $I(x)=x$ provides a power ratio representation for $\left(K \times K, \preccurlyeq_{m}\right)$ since $I=A \psi^{B}$ for $A=1 / \alpha$ and $B=1 / \beta$. When only a single attribute is considered, one need not use a power than 1.0 in the power ratio representation. Krantz and Shepard point out that powers other than 1.0 are required if the structure of crossmodality matching is included in the numerical representation. Their line of analysis has been omitted here, because it is not required for the development of the multiattribute generalization of the power law to which we now turn.

Let $K=K_{1} \times K_{2} \times \cdots \times K_{N}$, where each $K_{i}$ is a physically parametrized set. For example, suppose that each $K_{i}$ is a set of pure tones varying in intensity at some fixed frequency $\eta_{i}$. Any $x \in K$ is a complex acoustical stimulus with intensity $x_{i}$ at frequency $\eta_{i}$. If $F: K \rightarrow \mathrm{Re}^{+}$is any function, then we way that $F$ satisfies a multiattribute power law iff for every $i$, there exist functions $G_{i}: \bar{K}_{i} \rightarrow \mathrm{Re}^{+}$and $H_{i}: \bar{K}_{i} \rightarrow \operatorname{Re}^{+}$ such that

$$
F(x)=H_{i}\left(\bar{x}_{i}\right) x_{i}^{G_{i}\left(\bar{x}_{i}\right)}
$$

for all $x \in K$. By definition, $F$ satisfies a multiattribute power law whenever $F$ is a power function of the $i$ th attribute $K_{i}$ for any $i$ and any fixed choice $\bar{x}_{i}$ on the remaining attributes.

The relation (15) implies that $F$ is the exponential of a FOP function. To see this, note that (15) implies that

$$
\ln F(x)=G\left(\bar{x}_{i}\right)\left(\ln x_{i}\right)+\ln H_{i}\left(\bar{x}_{i}\right)
$$

for any $x \in K$. Let $L$ be the sequence of $N$ logarithmic transformations $L=(\ln , \ln , \ldots, \ln )$. Then $(16)$ asserts that $\ln F$ is multiaffine with respect to $L$, so by Lemma $2, \ln F=\bar{P} \cdot L$ or $F=e^{\bar{P} \cdot L}$ for some FOP $P$. It is easy to show that, conversely, if $F=e^{\bar{p} \cdot L}$ for some FOP $P$ and $L$ the sequence of logarithmic transformation, then $F$ satisfies a multiattribute power law (15). Thus, the multiattribute power law is equivalent to the assertion that $F=e^{\bar{p} \cdot L}$. 
The multiattribute power law will be axiomatized in terms of the formalism of subsection II(B). Let $\preccurlyeq_{m}$ be an empirical ordering of $K \times K$ determined, for example, by ratio estimation and assume as in Relation theory that $\left(K \times K, \preccurlyeq_{m}\right)$ satisfies the algebraic difference axioms. Thus, there exists a function $\psi: K \rightarrow \operatorname{Re}^{+}$that represents $\preccurlyeq_{m}$ as an ordering of numerical ratios. Let $K_{i x}$ be the set of all $\left.x\right|_{i} a$ for $a \in K_{i}$; let $\psi_{i x}$ be the restriction of $\psi$ to $K_{i x}$; let $\preccurlyeq_{i x}$ be the restriction of $\preccurlyeq_{m}$ to $K_{i x} \times K_{i x}$; let $\theta_{i x}: K_{i} \rightarrow K_{i x}$ be defined by $\theta_{i x}(a)=\left.x\right|_{i} a$; and let $\left(K_{i} \times K_{i}, \leqslant_{i x}^{*}\right)$ be the structure isomorphic to $\left(K_{i x} \times K_{i x}, \preccurlyeq_{i x}\right)$ under $\theta_{i x}$.

For any $i$ and $x, \psi_{i x} \cdot \theta_{i x}$ is a ratio representation for $\left(K_{i} \times K_{i}, \preccurlyeq_{i x}^{*}\right)$. The following axiom rephrases the uniattribute power ratio axiom (14) in terms of the uniattribute substructures $\left(K_{i} \times K_{i}, \preccurlyeq_{i x}^{*}\right)$.

Multiattribute Power Ratio Axiom. For every $i \in \mathbf{N}, x \in K, a, b \in K_{i}$ and positive real $t$,

$$
\left(\left.x\right|_{i} a\right)\left(\left.x\right|_{t} b\right) \sim_{m}\left(\left.x\right|_{t} t a\right)\left(\left.x\right|_{i} t b\right) .
$$

Since by definition of $\leqslant_{i x}^{*}$, (17) yields $a b \sim_{i x}^{*}(t a)(t b)$, the representation $\psi_{i x} \cdot \theta_{i x}$ must be a power function. Moreover, it is easy to show that if $\bar{x}_{i}=\bar{z}_{i}$ for any $z \in K$, then $\psi_{i x} \cdot \theta_{i x}=\psi_{i z} \cdot \theta_{i z}$. It follows that there are well-defined functions $G_{i}: \bar{K}_{i} \rightarrow \operatorname{Re}^{+}$and $H_{i}: \bar{K}_{i} \rightarrow \mathrm{Re}^{+}$satisfying

$$
\psi_{i x} \cdot \theta_{i x}(a)=H_{i}\left(\bar{x}_{i}\right) a^{G_{i}\left(\bar{x}_{i}\right)}
$$

for any $i \in \mathbf{N}, x \in K$ and $a \in K_{i}$. But note that by definition of $\psi_{i x}$ and $\theta_{i x}$, $\psi(x)=\psi_{i x} \cdot \theta_{i x}\left(x_{i}\right)$ for any $x \in K$. Combining this with (18) we have

$$
\psi(x)=H_{i}\left(\bar{x}_{i}\right) x_{i}^{\left(G_{i}\left(\bar{x}_{i}\right)\right.}
$$

for every $x \in K$ and $i \in \mathbf{N}$. Thus, $\psi$ satisfies a multiattribute power law. Therefore, there exists a FOP $P$ such that $\psi=e^{\bar{P} \cdot L}$ for $L=(\ln , \ln , \ldots, \ln )$.

We have shown that if $\left(K \times K, \preccurlyeq_{m}\right)$ satisfies the algebraic difference axioms and the multiattribute power ratio axiom, then it has a ratio representation $\psi$ satisfying a multiattribute power law (15) and $\psi=e^{\bar{P} \cdot L}$ for some FOP $P$ and the sequence $L$ of logarithmic transformations.

Stevens has been the foremost advocate of the hypothesis that the psychophysical law is a power law, and it may be appropriate to relate briefly the present theory to his work. The uniattribute power ratio axiom can fairly be read into his hypothesis that

there is a general psychophysical law relating subjective magnitude to stimulus magnitude, and ... this law is simply that equal stimulus ratios produce equal subjective ratios. (Stevens, 1957).

Stevens did not consider the possibility of a multiattribute power law (15) nor its relation to the exponential FOP $e^{\bar{P} \cdot L}$ for $L$ the sequence of logarithms, but he did point out that the power associated with particular attributes had been found empirically to vary with different contextual factors in a way that is suggestive of the 
multiattribute power law. He called this variation the operation of a power group (Stevens, 1966, 1971).

Luce has provided two derivations of multiattribute power law in the two attribute case. Luce (1959a, Sect. 2.C) derives a two attribute psychophysical function $\psi(x, y)$ satisfying a multiattribute power law from assumptions concerning the discriminability of stimuli. Luce $(1959 \mathrm{~b})$ shows that a proposed principle of theory construction forces $\psi(x, y)$ to satisfy a multiattribute power law if $x, y$ and $\psi(x, y)$ are all measured on ratio scales. In both derivations, an analysis of the partial derivatives of $\psi$ presented in Luce (1959a) is used to establish that $\psi$ has the form

$$
\Psi(x, y)=a x^{b} y^{c+d(\ln x)},
$$

where $a, b, c, d$ are real constants. Luce's solution is equivalent to a FOP represen tation, since

$$
a x^{b} y^{c+d(\ln x)}=e^{\ln a+b(\ln x)+c(\ln y)+d(\ln x)(\ln y)} .
$$

Although Luce's approach presumably generalizes to more than two variables, the derivation based on Lemmas 1 and 2 given here appears to be much simpler. The relationship between FOP representation theory, multiattribute psychophysics, and the invariance principles proposed in Luce $(1959 \mathrm{~b})$ deserves exploration, but this topic must be treated elsewhere. ${ }^{2}$

\section{CONCLUSION}

This essay has presented a number of FOP generalizations of standard measurement theories. These generalizations exhibit a logical pattern that is both a mathematical technique and a substantive strategy of theory construction. Before attempting to give an abstract characterization of this pattern, it will be useful to sketch the pattern in the context of the FOP generalization of EU theory.

Suppose $\left(L, K, \preccurlyeq_{p}\right)$ is a multiattribute EU structure, i.e., $K=K_{1} \times K_{2} \times \cdots \times K_{N}$ is a Cartesian product of attributes, $L$ is the set of lotteries based on $K$ and $\leqslant_{p}$ is a preference relation satisfying EU axioms. To achieve the FOP generalization of the utility representation, it was first necessary to identify uniattribute EU substructures $\left(L_{i}, K_{i}, \preccurlyeq_{i x}^{*}\right)$ which are isomorphically embedded within $\left(L, K, \preccurlyeq_{p}\right)$. These substructures are lotteries $L_{i}$ based on consequences in $K_{i}$ to be received along with the fixed consequence $\bar{x}_{i}$, with $\preccurlyeq_{i x}^{*}$ the preference relation induced by the restriction of $\preccurlyeq_{p}$ to $L_{i x}$, the isomorphic image of $L_{i}$.

The FOP utility representation is equivalent to the assumption that uniattribute utility independence holds, that is, that the preference relation $\leqslant_{i x}^{*}$ of any uniattribute EU substructure $\left(L_{i}, K_{i}, \preccurlyeq_{l x}^{*}\right)$ is invariant with respect to changes in the fixed conse-

\footnotetext{
${ }^{2}$ An investigation of this relationship would also have to take into account the material in Chap. 10 of Krantz et al. (1971) and Luce (1964). I am grateful to A. A. J. Marley for pointing out this last reference.
} 
quence $\bar{x}_{i}$. The technique of proof consists in showing that this assumption combines with the affine uniqueness of utility representations to imply that any utility function $U: K \rightarrow \operatorname{Re}$ for $\left(L, K, \preccurlyeq_{p}\right)$ must be multiaffine with respect to a sequence $\phi$ of uniattribute scales. Lemma 2 then implies the existence of a FOP $P$ such that $F=\bar{P} \cdot \phi$.

The substantive interpretation of the FOP utility representation is analogous to that of the FOP generalization of Relation theory. The fundamental empirical structure of EU theory is the preference relation over lotteries, and hence, the restricted preference relation $\preccurlyeq_{i x}^{*}$ of the EU substructure $\left(L_{i}, K_{i}, \preccurlyeq_{i x}^{*}\right)$ is the fundamental property of utility for the attribute $K_{i}$ in the context of the fixed consequences $\bar{x}_{i}$. The FOP generalization of utility theory is based on the assumption that the fundamental psychological property of uniattribute utility is invariant with respect to changes in the context of fixed consequences on the remaining attributes. The FOP utility representation thus embodies a psychological hypothesis that constituent uniattribute utilities of a multiattribute utility structure satisfy a kind of context invariance.

As argued in Section III, the FOP generalization of Relation theory is based on a postulate of context invariance, and an analogous postulate underlies the FOP components generalization of additive conjoint measurement. We end this essay with some metatheoretical comments that attempt to characterize in informal, albeit abstract, terms the logical pattern of FOP measurement theory.

The crucial prerequisite of a FOP axiomatization is a measurement structure that establishes the existence of an affinely unique scale $F: K \rightarrow \operatorname{Re}$ on some set $K$. Any such measurement structure identifies a set of fundamental empirical relations and properties of these relations (axioms) from which the existence and uniqueness of $F$ can be derived. If the basic set $K$ is now regarded as a Cartesian product, the measurement structure based on $K$ will contain many uniattribute substructures determined by fixing all but the $i$ th component at some context $\bar{x}_{i}$ and by restricting the fundamental empirical relations to elements of this form. To establish that the scale $F$ is multiaffine with respect to a sequence $\phi$ of scales, one must be able to prove, first, that every uniattribute substructure is itself an example of the measurement structure to be generalized, and second, that the fundamental relations of a uniattribute substructure are unaffected by changes in the context $\bar{x}_{i}$ in terms of which they are defined.

The key to the FOP generalization of a measurement theory lies in finding the right definition of uniattribute substructures and in formulating the appropriate property of context invariance. Once this is accomplished, one chooses for every $i$, a scale $\phi_{i}$ that represents the relations in an arbitrarily chosen substructure on the $i$ th attribute and then context invariance implies that $\phi_{i}$ represents the relations in any other uniattribute substructure on the $i$ th attribute. But since $F$ is a representation for the fundamental relations on $K$, the restriction of $F$ to any uniattribute substructure must also be a representation for the relations in that substructure. Hence, for any $i$, $F$ and $\phi_{i}$ both represent the fundamental relations in any uniattribute substructure on the $i$ th attribute, and it follows that $F$ is multiaffine with respect to the sequence $\phi=\left(\phi_{1}, \phi_{2}, \ldots, \phi_{N}\right)$ of scales. Lemma 2 can then be invoked to establish the FOP representation. Moreover, insofar as the fundamental empirical relations of a 
measurement theory are psychologically or behaviorally interpreted, context invariance, the key property of a FOP generalization, must constitute a strong constraint on the structure of behavior.

It should be clear that the strategy outlined here for developing FOP representations is not restricted to the substantive theoretical frameworks of EU theory or Relation theory. This is fortunate, since EU theory is well known to fail as a descriptive psychological theory and Relation theory might also turn out to be false. But insofar as a psychological theory postulates a measurement structure that implies the existence of an affinely unique scale, and such theories abound, this theory is a candidate for FOP generalization. The rationale for interest in FOP representations resides in the pattern of context invariance which they characterize. Given the ubiquity of affinely unique numerical representations in psychological theories, it is to be hoped that theoretically interesting FOP generalizations can be developed for many, if not all such theories.

\section{REFERENCES}

ACZEL, J. Lectures on functional equations and their applications. New York: Academic Press, 1966.

DYER, J. S., \& SARIN, R. K. Measurable multiattribute value functions. Operations Research, 1979. 27 (4), 810-822.

ELLSBERG, D. Classic and current notions of "measurable utility." Economic Journal, 1954, 64, $528-556$.

Fishburn, P. C., \& KeENEy, R. L. Seven independence concepts and continuous multiattribute utility functions. Journal of Mathematical Psychology, 1974, 11, 294-327.

Fishrurn, P. C., \& KeENEY, R. L. Generalized utility independence and some implications. Operations Research, 1975, 23 (5), 928-940.

Keeney, R. L. Quasi-separable utility functions. Naval Research Logistics Quarterly, 1968, 15. $551-565$.

KeENEY, R. L. Utility independence and preferences for multiattributed consequences. Operations Research, 1971, 19, 875-893.

KEENEY, R. L. Utility functions for multiattributed consequences. Management Science, 1972, 18 (5), 276-287.

Keeney, R. L., \& Raiffa, H. Decisions with multiple objectives: Preferences and value tradeoffs. New York: Wiley, 1976.

KRANTZ, D. H. A theory of magnitude estimation and cross-modality matching. Journal of Mathematical Psychology, 1972, 9, 168-199.

Krantz, D. H.. Luce, R. D., Suppes, P., \& Tversky, A. Foundations of measurement. New York: Academic Press, 1971.

LUCE, R. D. Individual choice behavior. New York: Wiley, 1959(a).

Luce, R. D. On the possible psychophysical laws. Psychological Review, 1959(b), 66, 81-95.

LuCE, R. D. A generalization of a thcorem of dimensional analysis. Journal of Mathematical Psychology, 1964, 1, 278-284.

LuCE, R. D., \& RaIfFA, H. An axiomatic treatment of utility. In R. D. Luce \& H. Raiffa, Games and decisions: Introduction and critical survey, New York: Wiley, 1957. Pp. 23-31.

Maclane, S., \& Birkhoff, G. Algebra. New York: Macmillan, 1967.

MArKs, L. E. On scales of sensation: Prolegomena to any future psychophysics that will be able to come forth as a science. Perception and Psychophysics, 1974, 16 (2), 358-376. 
RaIFfa, H. Preferences for multi-attributed alternatives. RM-5868-DOT/RC, The Rand Corporation, Santa Monica, Calif., 1969.

SHEPARD, R. N. Psychological relations and psychophysical scales: On the status of "direct" psychophysical measurement. Journal of Mathematical Psychology, 1981, 24, 21-57.

Stevens, S. S. On the psychophysical law. Psychological Review, 1957, 64, 153-181.

Stevens, S. S. Power-group transformations under glare, masking, and recruitment. Journal of the Acoustical Society of America, 39, 1966, 225-235.

STEvens, S. S. Issues in psychophysical measurement. Psychological Review, 1971, 78, 426-450.

Stevens, S. S. Psychophysics. New York: Wiley, 1975.

RECEIVED: March 31, 1983 